\title{
Kapitel 11 \\ Zukünftiges als Erzählfragment
}

\section{Hinführung}

Verweise auf Ereignisse, die zum Zeitpunkt des Sprechaktes noch nicht geschehen sind, konstituieren keine „Erzählungen,“ in dem Sinn, wie sie in Teil III dieser Arbeit untersucht wurden. Zugleich zeigte sich in der Analyse des paulinischen Erzählstils immer wieder, dass zentrale Merkmale der Narration im corpus Paulinum in auffälliger Häufung in Passagen mit Zukunftsbezug auftreten.

Anhand des Imperativs in Gal 4,12a - der als Aufforderung auf ein Handeln in der Zukunft, nach Erhalt des Briefes, ausgerichtet ist - wurde zudem bereits angedeutet, dass zukünftige Ereignisse durchaus von Bedeutung für „implizite Erzählungen," für Protoerzählungen, sind (Kapitel 9, Abschnitt 5.4.4). Wer über Zukünftige Geschehnisse spricht, bringt dadurch eine Ereignisfolge zum Ausdruck, die zu einem späteren Zeitpunkt als Erzählung tatsächlich geschehener Ereignisse in der Vergangenheit formuliert werden könnte, die in der Gegenwart also (noch) eine „potenzielle Erzählung“ ist. ${ }^{1}$

Diese Verschiebung des deiktischen Zentrums (siehe oben, Kapitel 6, Abschnitt 3.2.2) ist im Griechischen teilweise auch in der Grammatik verankert. Beispielsweise ist alleine schon aufgrund der Übernahme des Tempus in der indirekten Rede im Griechischen in der Ankündigung einer zukünftigen (Binnen-) Erzählung die spätere Erzählung präsent: ,Dann wird er antworten und sagen: ,...

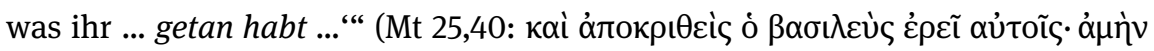

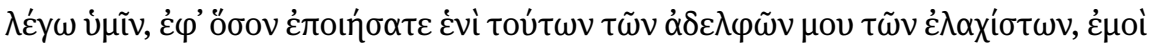
غ̇лoเท́ $\alpha \tau \varepsilon$ ). Außerdem kann im proleptischen Ind. Aor. „[e]twas, was sich (aus der Sicht des Sprechers/Schreibers) später bzw. nach etwas anderem virwirklichen wird ..., ... vorausgenommen und gleichsam als bereits verwirklicht dargestellt“ werden. ${ }^{2}$ Ähnliches gilt auch für den „epistolarischen“ Ind. Aor.: Einerseits kann es sich hier (1) um einen Fall simultaner Narration handeln (siehe dazu oben, Kapitel 6, Abschnitt 3.4.2.1). Die im Aorist erzählten Ereignisse können jedoch aus Sicht des Schreibers auch vollkommen in der Zukunft liegen, wenn etwa auf

1 Vgl. auch bereits Weinrich, „Narrative Theologie,“ 332 der die Zentralität des „hypothetischen Erzählens“ von noch nicht geschehenen Ereignissen für die Theologie betont: „Die Prophetien des biblischen Corpus können als erzählende Entwürfe noch nicht geschehener Handlungen angesehen werden, als ein Vor-Erzählen also.“

2 Vgl. GGNT 199k mit Verweis auf 1. Kor 7,28. Teilweise ist die Abgrenzung zum gnomischen Ind. Aor. nicht einfach (z. B.: 1. Kor 15,49). 
spätere Begleithandlungen zum Senden des Briefes verwiesen wird (vgl. etwa das „Senden“ von Mitarbeitern, das durch है $\pi \varepsilon \mu \psi \alpha$ ausgedrückt wird; z. B. Phil 2,28, Phlm 12; in den umstrittenen Paulusbriefen siehe Eph 6,22, Kol 4,8). Es handelt sich hierbei also streng genommen um (2) „prädiktive Narration“ (vgl. Kapitel 6, Abschnitt 3.2.3), wobei vom Standpunkt der den Brief in Empfang nehmenden Adressaten aus auf die Ereignisse zurückgeblickt wird.

Auch wo Zukünftiges nicht derart eindeutig aus einer hypothetischen futurischen Perspektive als bereits geschehen dargestellt wird, evoziert die bloße Nennung von futurischen Ereignissen oft als Protoerzählung die in der Gegenwart noch potenzielle Erzählung, die zu einem späteren Zeitpunkt auch explizit erfolgen könnte.

Dass die Simulation eines Erzählaktes in der Rede von noch ausstehenden Ereignissen oft nahezu unvermeidlich ist, zeigt aber eindrucksvoll auch Phil 2,16: In V. 14 fordert Paulus zu einem Lebenswandel ohne Murren und Zweifel auf. V. 15 expliziert in einem Finalsatz, welches Resultat ein solches Verhalten für die Philipper haben würde. V. 16a expliziert, wie all dies ${ }^{3}$ verwirklicht werden soll: „... indem ihr das Wort des Lebens festhaltet.“ Sehr prägnant fügt Paulus dann noch an, was dies als Folge für ihn bedeuten würde: „... mir zum Ruhm am Tag Christi“

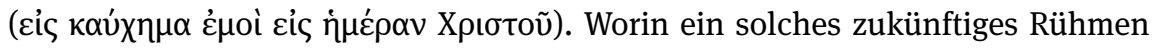
bestehen würde, ließe sich im Deutschen (und Englischen) stilistisch am angemessensten im Futur II zum Ausdruck bringen, also durch ein Tempus mit grammatikalisierter sekundärer Orientierungszeit: „... damit mein Ruhm sein wird, dass ich nicht vergeblich gelaufen sein oder gearbeitet haben werde.“ Paulus

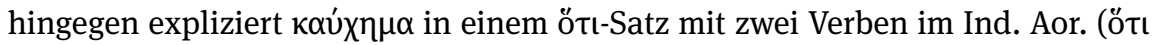

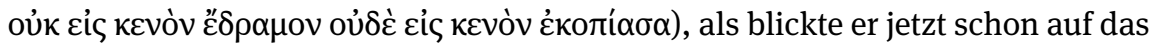
zurück, was nur dann am Tag des Herrn Teil seiner Vergangenheit sein wird, falls die Philipper die eben verfasste Ermahnung sich zu Herzen nehmen werden bzw. genommen haben werden.

\section{Beispiel 2. Thess 3,7b-8}

Die im Hinblick auf Diserzählungen bereits erwähnte (Kapitel 10, Abschnitt 6) Passage 2. Thess 3,7b-8 (siehe Abb. 54) ist auch in dieser Hinsicht aufschlussreich und soll daher im Folgenden kurz unter dem Gesichtspunkt der Rolle futurischer Ereignisse für die Identifizierung von Protonarrativen besprochen werden.

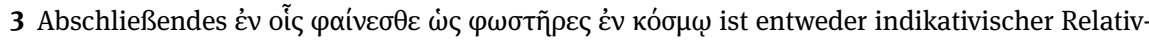
satz oder schließt in relativem Satzanschluss einen weiteren Imperativ an. 


\begin{tabular}{|c|c|c|c|}
\hline V. $7 b$ & 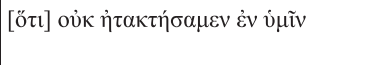 & $\begin{array}{l}\text {,[Denn] wir haben unter euch keinen unordentlichen } \\
\text { Wandel geführt.' }\end{array}$ & Ereignis (1') \\
\hline V. $8 \mathrm{a}$ & 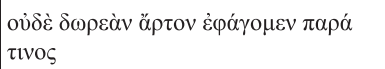 & $\begin{array}{l}\text {,Wir haben uns auch nicht umsonst vom Brot anderer } \\
\text { Leute ernährt. }\end{array}$ & Ereignis (2') \\
\hline V. $8 \mathrm{~b}$ & $\dot{\alpha} \lambda \lambda^{\prime}$ [erg. $\left.\alpha \rho^{\prime} \tau \nu \nu \dot{\varepsilon}_{\varphi} \alpha \gamma_{\gamma} \mu \varepsilon v\right]$ & ,Sondern [wir ernährten uns,] & Ereignis (2) \\
\hline V. $8 \mathrm{c}$ & 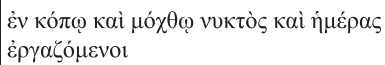 & ,indem wir Tag und Nacht angestrengt arbeiteten, ‘ & Ereignis (1) \\
\hline V. $8 \mathrm{~d}$ & 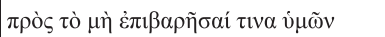 & ,um keinem von euch zur Last zu fallen. ‘ & Ziel ( $\left(2^{\prime}\right)$ \\
\hline
\end{tabular}

Abb. 54: Ereignis-Beziehungen in 2. Thess 3,7b-8.

Vergleicht man das hinter der Diserzählung stehende Protonarrativ $\left(1^{\prime}\right)+\left(2^{\prime}\right)$ mit die explizite Erzählung (2) + (1), so fällt auf, dass die sinnhafte Verknüpfung der Ereignisse etwas unterschiedlich gelagert ist, obwohl die ausgewählten Betrachtzeiten symmetrisch verlaufen (siehe hierzu Kapitel 12, Abschnitt 2.3.2): Für die Protoerzählung kann ein konsekutives Grund-FOLGE-Verhältnis rekonstruiert werden, ${ }^{4}$ in der expliziten Erzählung liegt eine modal-instrumentale Konnexion (ERGEBNIS-Mittel) vor. Beide Verbindungen sind eng miteinander verwandt (siehe oben, Kapitel 5, Abschnitt 3.4). Im ersten Fall wird die Apodosis des zugrundeliegenden Konditionalgefüges als das logische Resultat eines Geschehens dargestellt, ${ }^{5}$ im zweiten Fall wird anti-ikonisch von einer diesem Geschehen diametral entgegenstehenden Situation ausgehend das in der zugrundeliegenden Protasis enthaltene Handeln als Mittel dargestellt. ${ }^{6}$

Der Vorwurf, welcher im Protonarrativ $\left(1^{\prime}\right)+\left(2^{\prime}\right)$ enthalten ist, unterstellt somit kein Motiv, sondern fokussiert lediglich auf die Folgen eine bestimmten Vorgehens. Das ihm entgegengestellte explizite Narrativ, nimmt das Ende des Protonarrativs auf und stellt dem Ereignis (2') den eigentlichen „Schluss“ (2) der Erzählung entgegen. Dass in der Wirklichkeit Ereignis (2) - und nicht etwa (2') geschah, liegt aber nicht an irgendeinem Zufall, sondern daran, dass die Erzählfiguren bewusst ein an die Stelle von (1') tretendes Handeln wählten, ${ }^{7}$ nämlich

4 Zur Begründung des konsekutiven Verhältnisses siehe oben, Kapitel 10, Abschnitt 3.4.

5 D. h.: „Wenn man nicht selbst arbeitet, dann ist man für die Ernährung auf die Hilfe anderer Menschen angewiesen.“

6 D. h.: „Wenn man fleißig arbeitet, dann kann man sich selbst ernähren.“

7 Dieses intentionale Element ist in der modal-instrumentalen Konnexion nicht notwendigerweise erhalten, doch ist für den kooperativen Leser problemlos zu erschließen, dass die eigenständige Versorgung nicht ein zufälliges Begleitprodukt der eventuell mit anderem Zweck verfolgten 
Ereignis (1), das sich aus ihrer Sicht als Mittel zum Erreichen von Ereignis (2) eignen würde.

Der Text macht dieses zusätzliche intentionale Element in der expliziten Erzählung dadurch deutlich, dass das Ereignis (1) in V. 8c nicht alleine steht,

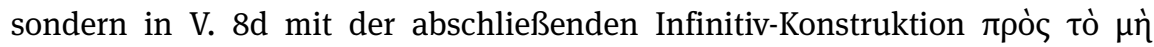

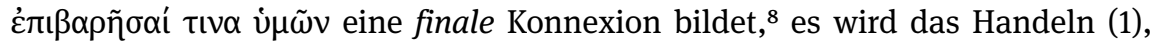
welches ein Mittel zum Erreichen von (2) ist, also zugleich als Mittel zum Vermeiden des Ziels (2') qualifiziert.

V. 8d expliziert folglich nochmals, was schon aus der Konnexion 8b-8c hervorgeht, dass nämlich innerhalb der dieser Miniaturerzählung korrespondierenden erzählten Welt weitere temporal und sinnhaft verknüpfte Elemente ergänzt werden müssen - (3) das (mentale) Ereignis eines bestimmten Wunsches, nämlich der Wunsch, (2') zu vermeiden, welcher wiederum zu einem Plan (4) führt, der nämlich darin besteht, (1) als Mittel zum Erreichen von (2) $=\left(\neg 2^{\prime}\right)$ einzusetzen, dessen tatsächliche Umsetzung dann in der Retrospektive in (1) + (2) explizit erzählt wird.

Doch die Infinitiv-Konstruktion impliziert nicht nur zwei weitere mentale Ereignisse innerhalb der Erzählung, sondern liefert noch eine weitere erzähltheoretisch bedeutsame Erkenntnis. Das Gegenstück zu dem aus (3) hervorgehenden aktiven Plan (4) und dem darauf basierenden Verhalten stellt, mit Ryan gesprochen (siehe Kapitel 10, Abschnitt 3), die passive Prognose (5) dar.

Mit anderen Worten, man kann an dieser Stelle der Chronologie ein weiteres „virtual embedded narrative“ postulieren: Damit die Erzählfiguren die Aktivität (1) als Mittel zum Vermeiden von ( $\neg$ 2') bzw. Erreichen von (2) planen können, müssen sie zunächst simulieren, was aus (1') folgen würde. Diese passive Simulation weist wiederum die Handlung $\left(1^{\prime}\right)+\left(2^{\prime}\right)$ auf. ${ }^{9}$ Die Erzählung impliziert also, dass in der erzählten Vergangenheit Paulus und seine Mitarbeiter bewusst darüber nachdachten, wozu das Unterlassen fleißigen Arbeitens, „Faulheit,“ führen würde.

(z.B. aus purer Arbeitswut oder Gewohnheit) Beschäftigung darstellt. Dies ergibt sich daraus, dass davon auszugehen ist, dass die Arbeit nicht umsonst als mühevoll charakterisiert wird. Entsprechend ist ein guter Grund dafür zu suchen, dass diese dennoch durchgeführt wurde. Auf eine finale Konnexion - welche das finale Element beinhalten würde - wird wohl verzichtet, um das Eintreten der zugrundeliegenden Apodosis explizit festzuhalten. Vgl. dann die finale Konstruktion in V. 8d.

8 GGNT 336a.

9 In dieser Prognose kommt die konditionale Basis selbst zum Vorschein: „Wenn wir X machen, dann wird Y geschehen.“ 
Es ergibt sich die äußerst interessante Konstellation, dass das Protonarrativ $\left(1^{\prime}\right)+\left(2^{\prime}\right)$ innerhalb dieses so kurzen Textes folglich auf zwei verschiedenen Ebenen auftaucht. Auf der einen Seite diserzählt es der Erzähler von 8b-8c in 7b-8a als Kontrastfolie zur eigenen expliziten Erzählung. Soweit könnte man noch die Vermutung anstellen, dass er für die mentale Simulation des Erzählaktes die Perspektive von Gegnern eingenommen hätte (also im Sinne eines Vorwurfs nach

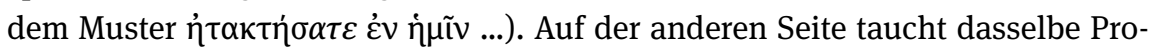
tonarrativ - nun sprachlich verdichtet auf das Fragment eines einzelnes Verbs

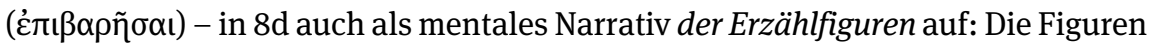
nehmen in der Vergangenheit mental einen zukünftigen Standpunkt ein, von dem aus betrachtet es ihnen wichtig ist, dass man die potenzielle Erzählung $\left(1^{\prime}\right)+\left(2^{\prime}\right)$ im Rückblick eben nicht über sie wird erzählen können. Dass (2) intentional auf (1) bezogen ist und zusätzlich als Mittel zum Zweck des Vermeidens des غ̇лıß $\alpha \tilde{\eta} \sigma \alpha \iota ~\left(\neg 2^{\prime}\right)$ charakterisiert wird, weist daher nach, dass auch innerhalb der Erzählung ein Protonarrativ (für das erzählte Wir) vorausgesetzt wird.

Man könnte mit Ryan von einem sich in der erzählten Welt nicht erfüllenden, „virtuellen“ eingebetteten Narrativ sprechen - oder eben von einem ProtoBinnennarrativ, also einer Erzählung, die als mentales Narrativ von Erzählfiguren in eine Rahmenerzählung eingebettet ist, ohne im Rahmen dieser Erzählung tatsächlich verbalisiert zu werden.

Der Beispieltext 2. Thess 3,7b-8 ist in zweifacher Hinsicht hilfreich, um die Beschäftigung mit Zukunftsbezügen im Rahmen impliziter Erzählungen einzuleiten. Erstens demonstriert er eindrücklich, dass es möglich ist, auf dichtem Raum durch Verweise auf zukünftige Ereignisse Handlungszusammenhänge anzudeuten, die das Potenzial einer Erzählung haben, auch wenn sie zum gegenwärtigen Zeitpunkt noch nicht erzählt werden können.

Der Text demonstriert aber nicht nur die Relevanz dieser großen Kategorie als Ganzer, sondern betont zweitens auch die Notwendigkeit, dass innerhalb der Kategorie der „zukünftigen Ereignisse“ genauer differenziert werden muss: „Zukünftig“ sind die ins Auge gefassten Ereignisse hier ja nur für die Erzählfiguren. Das Protonarrativ erscheint also als eingebettete Erzählebene innerhalb der Erzählung. Der Erzähler hingegen blickt nicht nur auf die mentale Ausbildung des Protonarrativs, sondern auch auf die eingetretenen und nicht eingetretenen Ereignisse zurück, manche der vormals potenziellen Erzählungen haben sich also mittlerweile als kontrafaktisch (lediglich „,virtuell“) herausgestellt, andere können nun explizit erzählt werden. Zugleich könnte er natürlich auch jederzeit selbst über Ereignisse sprechen (und tut dies auch direkt im Anschluss in 2. Thess 3,12), die auch aus Sicht des erzählenden Ichs/Wirs noch ausstehen. Für die Beschäftigung mit dieser Art an Ereignisbezügen muss daher im Folgenden ein belastbares Raster erarbeitet werden, welches die verschiedenen Optionen einzufangen weiß. 


\section{Zur Terminologie: Prolepse und Vorausdeutungen}

\subsection{Vorschläge}

Wenn im Hinblick auf Erzählungen von zukünftigen Ereignissen die Rede ist, so wird in diesem Zusammenhang in der Regel mit zwei Konzepten gearbeitet: dem der ,Prolepse“ von Genette und dem der ,Vorausdeutung“ von Lämmert. Der Umgang in der Fachliteratur mit diesen Kategorien ist leider nicht einheitlich.

So lässt sich einerseits bei vielen Autoren ein Gebrauch der beiden Lexeme als Synonyme feststellen. ${ }^{10}$ Andere Autoren bestehen auf einer Unterscheidung: So schreibt Fröhlich, bei Vorausdeutung sei lediglich an „Andeutung“ gedacht und nicht an tatsächliche Vorwegnahme. ${ }^{11}$ Zum Unterscheidungskriterium wird dann also die (1) Deutlichkeit des Zukunftsbezuges. Allerdings übersieht Fröhlich damit erstens, dass Lämmert durchaus die Möglichkeit einräumt, dass Vorausdeutungen (wenn auch in der Minderheit der Fälle) „bestimmte [d.h. in diesem Kontext: detaillierte ${ }^{12}$ Auskünfte über die Zukunft“ geben können ${ }^{13}$ und zweitens, dass Genette unter dem Terminus „Vorhalte“ (amorces; in Abgrenzung zu „Vorgriffen“) eben diese subtilen Anspielungen wiederum unter der Überschrift der Prolepse ebenfalls ausführlich diskutiert. ${ }^{14}$

Demgegenüber gestehen etwa Köppe und Kindt zu, dass auch die Prolepse durchaus „in der Form vager Hinweise erfolgen“ könne. ${ }^{15}$ Allerdings gehen auch diese Narratologen nicht von Deckungsgleichheit zwischen Genettes und Lämmerts Konzepten aus. Sie bestehen vielmehr darauf, dass im Fall der Prolepse gilt, dass sich das, worüber in einer Erzählung ,vorausblickend berichtet wird, in der erzählten Welt auch tatsächlich ... zutragen wird." ${ }^{16}$ Gerade darin sehen sie jedoch eine Abgrenzung zu Lämmerts Kategorie: Es sei irreführend, den Ausdruck Prolepse über dessen Beschreibung der Vorausdeutung zu erläutern: „Prolepsen

10 Martínez und Scheffel, Einführung, 35-36.

11 Fröhlich, Cliffhanger, 115,

12 Mit „bestimmt“ ist hier nicht die Gewissheit gemeint, mit welcher die Aussage gemacht wird, sondern der Grad an Detailliertheit. Dies zeigt auch eindeutig Lämmert, Bauformen, 142: „Noch einmal muß aber betont werden, daß zukunftsgewisse Voraussage nicht gleichzeitig bestimmte Aussage sein muß. Gerade hier ist dem Erzähler der weiteste Spielraum gegeben zwischen eindeutiger Ansage des Tatbestandes und dunkel-allgemeiner Ankündigung künftigen Unheils oder Glücks.“

13 Lämmert, Bauformen, 141.

14 Genette, Erzählung, 45-46.

15 Köppe und Kindt, Erzähltheorie, 188.

16 Köppe und Kindt, Erzähltheorie, 188. Hervorhebung nicht im Original. 
stellen Vorwegnahmen dar, aber keine Vorausdeutungen!"17 Auch diese Demarkation, die sich (2) an der Sicherheit des Eintretens der Zukunftsbezüge orientiert, ist jedoch letztlich nicht trennscharf. Denn Lämmerts Unterkategorie der ,zukunftsgewissen Vorausdeutung “18 scheint dieses Kriterium ebenfalls zu erfüllen.

Wollte man dennoch an einer Unterscheidung festhalten, so könnte man höchstens erwägen, die Bezeichnung „Prolepse“ lediglich für chronologisch vorgezogenes Erzählen zu verwenden, also wenn tatsächlich ein Teil der Erzählung früher erfolgt, als dies aufgrund der Chronologie des Erzählten zu erwarten wäre, und an jener Stelle dafür später diese Ereignisse übergangen werden. Demgegenüber könnte man die ,Vorausdeutung“ beschränken auf meta-narrative Kommentare, in welchen der Erzähler auf später erst zu erzählende Ereignisse hinweist, dadurch aber nichts im eigentlichen Sinn vorgezogen wird, und auf Sonderfälle des repetitiven Erzählens, in denen der Erzähler sich zwar nicht direkt an den Leser wendet, ein Ereignis jedoch mehrfach - zunächst außerhalb der chronologischen Folge - aufgenommen wird.

Auch wenn eine solche Unterscheidung - d. h. als Differenzierung (3) zwischen einem Phänomen der Ordnung auf der einen Seite und der Frequenz auf der anderen Seite - durchaus sinnvoll ist, bleibt jedoch fraglich, ob sie an der Begrifflichkeit „Prolepse vs. Vorausdeutung“ festgemacht werden sollte, da dies zumindest nicht im Sinne der Erfinder wäre: Denn Genette spricht einerseits von der „repetitiven Prolepse,“ welche keine spätere Ellipse ersetzt, sondern „im Voraus auf eine Ereignis Bezug [nimmt], das zu seiner Zeit in aller Breite erzählt wird.“19 Und Lämmert verweist andererseits etwa auf den Fall der „Vorausdeutung der Endsituation,“ wobei ein isoliert betrachtet offen erscheinendes Ende durch Vorausdeutungen bereits soweit ,abgesteckt“20 ist, dass es nicht noch explizit erzählt zu werden braucht. ${ }^{21}$

\subsection{Versuch einer Synthese}

Diese Überlappungen zeigen an, dass eine konstruktivere Inbeziehungsetzung angezeigt ist. Dabei ist hilfreich, was Genette an anderer Stelle (im Kapitel zur „Stimme“) über die „Zeit der Narration“ schreibt:22 Hier bemerkt er, dass es zwar einerseits „sich von selbst zu verstehen [scheint], dass die Narration nur nach

17 Köppe und Kindt, Erzähltheorie, 188.

18 Lämmert, Bauformen, 143-174.

19 Genette, Erzählung, 44.

20 Lämmert, Bauformen, 157.

21 Lämmert, Bauformen, 154-158.

22 Vgl. hierzu bereits oben, Kapitel 6, Abschnitt 3.2.3. 


\begin{tabular}{|l|l|l|}
\hline $\begin{array}{l}\text { Lämmert } \\
\text { Zukunftsungewisse } \\
\begin{array}{l}\text { Vorausdeutung } \\
\text { (einer Erzählfigur) }\end{array}\end{array}$ & $\begin{array}{l}\text { Prädiktive Narration } \\
\text { (einer Binnenerzählung) }\end{array}$ & $\begin{array}{l}\text { Zuläuterung } \\
\text { Zukunftsbezug innerhalb der erzählten Welt } \\
\text { aus der Perspektive der Erzählfigur }\end{array}$ \\
\hline $\begin{array}{l}\text { Zukunftsgewisse } \\
\text { Vorausdeutung }\end{array}$ & Prolepse & $\begin{array}{l}\text { Phänomen des Erzählens der Rahmenerzählung. } \\
\text { Gehört zur: } \\
\text { (a) Ordnung des Erzählens (falls es an der } \\
\text { entsprechenden chronologischen Stelle zu einer } \\
\text { Ellipse kommt) } \\
\text { (b) Frequenz des Erzählens (als Spezialfall des } \\
\text { repetitiven Erzählens, falls es zu keiner Ellipse } \\
\text { kommt) }\end{array}$ \\
\hline
\end{tabular}

Abb. 55: Zusammenschau der Kategorien von Lämmert und Genette.

dem kommen kann, was sie erzählt,“ dass aber andererseits auch Beispiele für „prädiktive Erzählung“23 beziehungsweise „frühere Narration“24 existierten. Dieses „Erzählen“ (das im Rahmen dieser Arbeit nicht als eigentlicher Erzählakt angesehen wird; vgl. oben, Kapitel 5, Abschnitt 3.2.3) geschehe normalerweise im Futur ${ }^{25}$ und trete in der Literatur nahezu ausschließlich in Binnenerzählungen auf, ${ }^{26}$ also wenn eine Erzählfigur über die Zukunft der erzählten Welt spricht. Selbst in Zukunftsromanen sei „die narrative Instanz fast immer vordatiert, liegt also noch weiter in der Zukunft als die in der Zukunft spielende Geschichte. “27 Auch der briefliche Aorist nimmt eine solche Position ein, von der auf die „weniger zukünftige“ Zukunft „,zurück“ geblickt werden kann.

Eine gute Deckungsgleichheit der Konzeptionen erhält man nun (vgl. Abb. 55), wenn man die prädiktiven Binnenerzählungen von Genette mit Lämmerts ,zukunftsungewissen Vorausdeutungen“28 von Erzählfiguren in Verbindung setzt. Diese Art der Vorausdeutungen postuliert Ereignisse für Zeitpunkte, die im Augenblick des Erzählaktes (der jeweiligen Erzählebene!) noch in der Zukunft liegen. Damit lassen sie sich abgrenzen von den verschiedenen Varianten an Prolepsen bzw. zukunftsgewissen Vorausdeutungen, in welchen über die Gegenwart der Basiserzählung ${ }^{29}$

23 Genette, Erzählung, 140. Anknüpfend an Todorov, Grammaire, 48.

24 Genette, Erzähltheorie, 141.

25 Genette, Erzähltheorie, 141.

26 Genette, Erzähltheorie, 142.

27 Genette, Erzähltheorie, 142.

28 Lämmert, Bauformen, 175-192.

29 Siehe dazu Köppe und Kindt, Erzähltheorie, 187. Das Festlegen einer Basiserzählung ist notwendig, damit sinnvoll von einer „Vorwegnahme“ gesprochen werden kann. Teilweise ist nicht 
hinausgegangen wird (jedoch nicht über den Standort des Erzählers der Rahmenerzählung hinaus). ${ }^{30}$

\section{Anwendbarkeit auf Protonarrative}

Geht man von dieser Systematik aus, ist natürlich noch immer die Frage zu klären, wie sie auf Protonarrative angewendet werden kann. In dieser Hinsicht ist es erhellend, Lämmerts Unterteilung vorausdeutender Motive näher zu betrachten: Grundsätzlich unterscheidet er zwischen zwei Wegen, ,auf denen der Mensch ... sich der Zukunft zu versichern“ suche: den der passiven Zukunftseinsicht und den der aktiven Zukunftsgestaltung. ${ }^{31}$

Der ersten Kategorie gehörten die Deutung von Träumen an, als untrüglich eingeschätzte Ahnungen, Prophetie und Weissagung. ${ }^{32}$ Das Vermögen des Menschen, auf diesem Wege Einsicht in die Zukunft zu erlangen, beurteilt Lämmert als „von Natur aus fragwürdig,“ da spätestens im Schritte der Auslegung angeblich übernatürlicher Einblicke menschlicher Irrtum ins Spiel komme. ${ }^{33}$ Wahre Vorsehung bleibe daher „Gottes Sache.“34 Was zweitens das Gestalten der Zukunft angeht, zählt Lämmert „Handeln, Sinnen und Trachten“ sowie ,auf vorausweisende Motive enger eingegrenzt ... Pläne, Entschlüsse, Anordnungen und Verabredungen“ auf. ${ }^{35}$ Eine vermittelnde Position zwischen passiver Einsicht in die Zukunft und aktiver Gestaltung derselben sieht Lämmert in den Kategorien „Wunsch“ und „Angst“ gegeben. Sie speisten sich aus unsicherer Zukunftseinsicht und motivierten zur Handlung. ${ }^{36}$

Lämmerts Zusammenstellung an vorausdeutenden Motiven ist hilfreich, da sie eine Klassifikation verschiedener Bezüge auf die Zukunft durch Erzählfiguren erlaubt. Besonders wichtig ist für die hier verfolgte Fragestellung jedoch auch,

\footnotetext{
klar, ob eine Basiserzählung mit Prolepsen angereichert ist, oder ob diese Passagen vielmehr selbst die Basiserzählung konstituieren - welche dann im Umkehrschluss mit Analepsen (d.h. der andernfalls als Basiserzählung gesetzten Ereignisse) ausgestattet wäre.

30 Lämmert, Bauformen, 142. Die Prolepsen können also bis hin zur Gegenwart des Erzählers reichen. Vgl. Genette, Erzählung, 41. Siehe auch Genette, Erzähltheorie, 40 für Beispiele von Ich-Erzählern, die auf die Situation der Abfassung verweisen.
}

31 Lämmert, Bauformen, 176.

32 Lämmert, Bauformen, 177.

33 Lämmert, Bauformen, 177.

34 Lämmert, Bauformen, 177.

35 Lämmert, Bauformen, 177.

36 Lämmert, Bauformen, 177. 
was Lämmert über die „erzählerische Wirkung auf den Leser, “ welche die Wirkung auf die Handlung der Erzählung selbst weit übersteigen kann, schreibt: ${ }^{37}$

Wo Träume, Ahnungen, Prophezeiungen im Laufe des erzählten Geschehens kundgetan werden, da steuern sie merkwürdigerweise trotz ihrer theoretischen Unverbindlichkeit den Leser in einer ähnlichen Weise wie es durch die gewissen Vorausdeutungen des Erzählers geschieht. Und das nicht nur, wenn die handelnde Person die Prophetie selbst ernst nimmt! ... Denn mag der junge Helmbrecht die Träume seines Vaters auch hoffärtig abtun, dem Zuhörer malen sie an Ort und Stelle bereits in dunklem Umriß das schmähliche Ende des Helden aus. Und welcher Hörer erwartete nicht mit Sicherheit das Eintreffen und die Folgen jener Verwünschung, die über der Wiege Dornröschens ausgesprochen wird, und bangte nicht fortan um die Lösung eines Zaubers, der sich viele Jahre später überhaupt erst realisiert?

Selbst Vorausdeutungen, die innerhalb der erzählten Welt keine nennenswerte Reaktion hervorrufen, können also „den Leser eine künftige Handlungsphase oder den Gesamtausgang mehr oder minder bestimmt antizipieren“"38 lassen. Diese Wirkung kann vom Erzähler natürlich auch ausgenutzt werden, um „trügerische Vorspiegelung“ auszulösen, ${ }^{39}$ also subtil unzuverlässig zu erzählen (siehe oben, Kapitel 3, Abschnitt 5.4).

Sowohl bei geglückter als auch gewollt torpedierter Prognose des Erzählfortgangs auf Leserseite spielen zusätzlich zur Handlung der expliziten Erzählung also auch noch zwei weitere Narrative eine Rolle: erstens das eingebettete mentale Narrativ der Erzählfigur, welche durch Annahmen über ihre Zukunft Aufschluss über ein zugrundeliegendes Protonarrativ gibt, und zweitens das durch die Leserlenkung evozierte Protonarrativ auf Seiten der Rezipienten. Dabei kann sich sowohl das Protonarrativ der Erzählfiguren als ausschließlich „virtuell,“ also innerhalb der Erzählwelt nicht bewahrheitend (vgl. Kapitel 10, Abschnitt 3), herausstellen, wie auch der von den Lesern vermutete Erzählfortgang ein mentales Narrativ konstituieren mag, welches letztlich gerade nicht dem Plot der eigentlichen Erzählung entspricht.

\section{Erweiterungen}

\subsection{Zukunftsgewisse Vorausdeutungen des Erzählers der Rahmenerzählung}

Die vorangehenden Überlegungen erlauben einen konstruktiven Umgang mit zukünftigen Ereignissen im Sinne von Erzählfragmenten von größeren

37 Lämmert, Bauformen, 178. Hervorhebung nicht im Original.

38 Lämmert, Bauformen, 179.

39 Lämmert, Bauformen, 186. 
Protonarrativen. Bevor diese Überlegungen auf die Paulusbriefe angewendet werden können, müssen allerdings noch drei Ergänzungen vorgenommen werden.

Gerade die von Lämmert erarbeitete lesersteuernde Wirkung der Vorausdeutungen des Erzählers scheint auch eine Korrektur seines Entwurfs an anderer Stelle zu erfordern. In der Analyse von Texten wie den Paulusbriefen macht es wenig Sinn, aufgrund des oben genannten Postulats der Unmöglichkeit wahrer Zukunftseinsicht die Kategorie der zukunftsgewissen Vorausdeutung des Erzählers nur auf dessen Vergangenheit zu beziehen. Wenn der Autor aufgrund einer Offenbarung zu sprechen meint, dann kommt dies einem Nacherzählen einer Handlung gleich, die ihm oder ihr zuvor von einem tatsächlichen zukünftigen Standpunkt aus erzählt bzw. sogar „live“ gezeigt wurde. Anders als in der „christlichen Dichtung“40 liegt in solchen „faktualen prädiktiven Erzählungen“ - wie man sie nennen könnte - kein Stilmittel zur Beglaubigung der letztlich lediglich zukunftsungewissen Vorausdeutungen von Erzählfiguren vor, sondern im Verständnis des Erzählenden selbst durchaus eine Prolepse auf den tatsächlichen Handlungsfortlauf der erzählten und zugleich realen Welt. Der Leser wird also nicht nur aufgefordert, Protonarrative zu bilden, sondern bekommt durch diese Art der zukunftsgewissen Vorausdeutung auch narrative Fixpunkte für diese Konstruktion mitgegeben.

Das bedeutet freilich nicht, dass eine Aussage über die Zukunft des Erzählers nur dann als „,zukunftsgewiss“ beurteilt werden kann, wenn sie in der realen Welt später auch tatsächlich eintritt (bzw. vom späteren Standpunkt aus: eingetreten ist). Entscheidend ist, ob das Ereignis aus Sicht des Erzählers fester Bestandteil der im Protonarrativ erzählten Welt ist oder ob es darin lediglich einen möglichen Handlungsfortgang markiert.

\subsection{Zukunftsgewisse Vorausdeutungen durch Binnenerzählungen von Erzählfiguren}

Diese Feststellung ist auch wichtig für den Umgang mit Äußerungen von Erzählfiguren. Denn wenn es zukunftsgewisses Erzählen über die Zukunft eines Erzählers, also auf der Ebene der Rahmenerzählung, geben kann, dann gilt dies selbstverständlich auch für die Erzähler von Binnenerzählungen. Auch Erzählfiguren können zukunftsgewisse Überzeugungen bezüglich der Zukunft der erzählten Welt ausbilden (z. B. aufgrund von erzählten Offenbarungen oder - eher in literarischen Werken üblich - aufgrund einer metaleptischen Information durch den Erzähler).

40 Lämmert, Bauformen, 180. 
Äußern sie diese Erwartung, so kommt auch dies dem Postulat einer zu einem späteren Zeitpunkt erzählbaren tatsächlichen Erzählung gleich. Auch hier kann das Kriterium für „Zukunftsgewissheit“ nicht sein, ob das angekündigte Ereignis „tatsächlich“ (d.h. in diesem Kontext: innerhalb der Rahmenerzählung) eintritt. ${ }^{41}$ Vielmehr muss auch hier beurteilt werden, ob es sich aus der Perspektive der Erzählfigur um einen Akt des prädiktiven Erzählens und damit um eine echte Prolepse handelt.

\subsection{Zukunftsungewisse Vorausdeutungen des Erzählers der Rahmenerzählung}

Eine weitere Kategorie, die bei Lämmert zu fehlen scheint, ist die der zukunftsungewissen Vorausdeutung des Erzählers. Betrachtet man Lämmerts Erläuterungen, könnte man auch den Eindruck bekommen, dass es diese prinzipiell gar nicht geben könne: Schließlich betont er, zukunftsungewisse Vorausdeutungen seien (anders als seine zukunftsgewissen Vorausdeutungen) gerade „nicht Zutat des Erzählers,“ sondern Gegenstand der Erzählung.42 Die „Zukunftsträume, Weissagungen und Mutmaßungen, aber auch Beschlüsse, Befehle und Pläne“ ${ }^{* 33}$ gehören eben den „handelnde[n] Personen.“ ${ }^{44}$

Nun wurde im letzten Abschnitt bereits in Frage gestellt, ob tatsächlich nur derjenige Erzähler (in der Eigenbeurteilung) zukunftsgewisse Vorausdeutungen vornehmen kann, der „seinen Standort außerhalb der Handlungsgegenwart hat. “ ${ }^{\circ 5}$ Sehr viel mehr muss allerdings noch die Gültigkeit des Umkehrschlusses bezweifelt werden.

Denn selbst für (1) fiktionale Erzählungen (mit oder ohne fiktivem Erzähler) gilt, dass der erzählende Autor im Moment des Niederschreibens (und in Abhängigkeit davon die eventuell vorliegende Erzählfigur des fiktiven Erzählers) ${ }^{46}$ über

\footnotetext{
41 Ist dies nicht der Fall, bedeutet dies außerdem nicht notwendigerweise, dass ein eventueller Erzähler der Rahmenerzählung sich dadurch als „täuschend unzuverlässig“ herausstellt (vgl. zur Definition Köppe und Kindt, Erzähltheorie, 237-244). Dies wäre nur dann der Fall, wenn ein solcher übergeordneter Erzähler den Lesern die Äußerungen einer Figur über zukünftige Ereignisse als zukunftsgewisse prädiktive Binnenerzählung vorstellen würde, sich dann später aber herausstellen würde, dass die Figur nie mit dieser Bestimmtheit über besagte Ereignisse gesprochen hätte.

42 Lämmert, Bauformen, 176.

43 Lämmert, Bauformen, 176.

44 Lämmert, Bauformen, 176.

45 Lämmert, Bauformen, 142.

46 Siehe oben, Kapitel 3, Abschnitt 5.2 und 5.4. Eine fiktionale Erzählung weist nur dann einen fiktiven Erzähler auf, wenn sie zur Vorstellung einlädt, dass die Erzählung von einer Erzählfigur erzählt wird. Ansonsten ist schlicht der Autor der Erzähler der Rahmenerzählung.
} 
die noch zu erzählende Zukunft spekulieren kann - über deren Ausgestaltung er oder sie zu diesem Zeitpunkt aber eventuell noch gar keine Entscheidung getroffen hat. Dies ist nicht nur eine theoretische Möglichkeit, sondern entspricht dem Vorgehen vieler Schriftsteller.

Umso mehr ist mit dieser Kategorie natürlich bei (2) faktualen Erzählungen zu rechnen, in welchen der Erzähler (sofern seine Person Gegenstand der Erzählung ist) lediglich durch Handlungen und daher auch nur sehr bedingt Einfluss auf die ihm noch bevorstehende Zukunft nehmen kann. Anders als in der fiktionalen Erzählung kann er oder sie nicht - im Rahmen der durch den Autor geschaffenen Gesetzmäßigkeiten - frei walten.

Daher sind für eine Analyse der paulinischen Protoerzählungen gerade auch die zukunftsungewissen Vorausdeutungen des Erzählers wichtig. In ihnen skizziert Paulus die Handlung einer Erzählung, die er so noch nicht - weder rückblickend noch „prädiktiv“ - mit Gewissheit erzählen kann. Je nachdem, wie er auf die aus seiner Sicht noch zukünftigen potenziellen Ereignisse Bezug nimmt (z. B. als Hoffnung oder als Befürchtung), wird die Verwirklichung dieses Protonarrativs als wünschenswert oder zu vermeiden qualifiziert. Es ist daher sicherlich $\mathrm{zu}$ erwarten, dass eine Kommunikationsintention, die auf ein (umzusetzendes oder zu vermeidendes) Handeln der Adressaten abzielt, von diesem Mittel Gebrauch macht (vgl. oben zur Kommunikationsintention; Kapitel 8, Abschnitt 4).

\section{Zukunftsgewisse und -ungewisse Vorausdeutungen auf die Vergangenheit des Erzählers}

\subsection{Hinführung}

Da der Rest der Arbeit an Protoerzählungen des Paulus interessiert ist, wird in den unten gebotenen Analysen bestimmter Ausdrucksformen für zukunftsgewisse und -ungewisse Vorausdeutungen der Schwerpunkt auf Verweise gelegt, die sich auch aus Sicht des Erzählers noch in der Zukunft befinden. Dabei kann es sich natürlich durchaus um Hinweise handeln, die Paulus einer Erzählfigur zuschreibt. Nicht weiter thematisiert werden sollen jedoch Vorausdeutungen von Erzählfiguren und des Erzählers, die sich auf einen Zeitpunkt innerhalb des Erzählten beziehen und aus Sicht des Paulus bereits in der Vergangenheit liegen.

Es handelt sich hierbei nämlich letztlich um ein Phänomen des klassisch zurückblickenden Erzählens. Meist wird einer Figur für einen bestimmten Zeitpunkt in der erzählten Vergangenheit die Ausbildung eines futurischen mentalen Narrativs zugeschrieben. Auch wenn dieses noch vorausschaut und damit eine lediglich potenzielle Protoerzählung darstellt, ist dieser Akt des Protoerzählens 
natürlich selbst ein tatsächlich erzähltes Ereignis - beziehungsweise ein Ereignis, das im Rahmen der erzählten Welt aufgrund anderer Aussagen angenommen werden muss. Es handelt sich hierbei also um narratologische Kategorien, die in der Beschreibung von gewöhnlichen Erzählungen eine Rolle spielen können und gerade bei gehaltvolleren Erzählungen und Erzählwerken, in welchen die Motivation der Figuren immer auch auf irgendeine Weise thematisiert sein wird, auch spielen muss.

Nicht notwendig für gehaltvolle Erzählungen, aber besonders typisch für diese sind zudem Vorausdeutungen des Erzählers selbst, die entweder in metanarrativen Kommentaren des Erzählers (z. B.: „Wir werden noch sehen, wie dies zu seinem Untergang führen wird.“) oder subtileren Anzeichen seiner Perspektive (z.B. in Charakterisierungen, die keiner Erzählfigur als Fokalisierungsinstanz zugeordnet werden können) zum Ausdruck kommen. ${ }^{47}$

Auch bei Paulus sind entsprechend Vorausdeutungen auf seine eigene Vergangenheit aus narratologischer Perspektive $\mathrm{zu}$ berücksichtigen - allerdings eigentlich unter der Überschrift der expliziten Erzählungen. Oben (Teil II) wurde auf eine entsprechende Diskussion verzichtet, da dort die Kategorien von Lämmert, Ryan und Genette noch nicht eingeführt worden waren.

Für entsprechende Analysen sind natürlich dann nicht nur die unten (Kapitel 13 und 14) angeführten Ausdrucksmöglichkeiten für Zukunftsbezug aus Sprecherperspektive zu berücksichtigen, sondern auch die verschiedenen Möglichkeiten, in der Koine eine „Zukunft in der Vergangenheit“ $\mathrm{zu}$ formulieren. ${ }^{48}$ Man vergleiche etwa Joh 7,39: „Dies aber sagte er über den Geist, den die an ihn

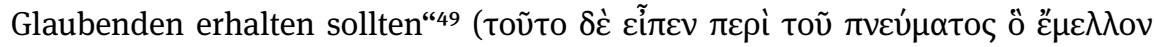

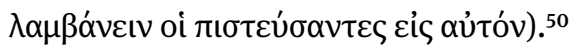

$\mathrm{Zu}$ beachten ist außerdem, dass Vorausdeutungen natürlich nicht immer durch komplexe lesersteuernde Motive zu erklären sind. Wie oben (Kapitel 8, Abschnitt 3.2) bereits am Beispiel von Gal 1,18 erläutert, kann bei entsprechender

47 Vgl. de Jonge, Narratology, 56-60 für Beispiele. Siehe grundsätzlich oben, Kapitel 3, Abschnitt 4.3 .

48 Für eine Übersicht siehe GGNT 203d. Kaiserzeitlich steht auch einfach ein „erzählendes Futur“: „Vom Standpunkt einer frühern Vergangenheit, auf den sich der Schriftsteller stellt, ist die der Gegenwart nähere, jüngere Vergangenheit zukünftig.“ So Schwyzer-Debrunner II, 293 mit Verweis auf Aelianus, Nat. an. 16.3. Auf das präsentisch formulierte Schießen auf das Yak ( $\beta \alpha \dot{\alpha} \lambda \lambda \varepsilon$ เ

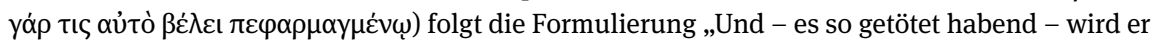

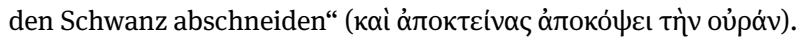

49 Vgl. Duden 1842.

50 Für Imperfekt von $\mu \varepsilon \dot{\lambda} \lambda \omega$ mit Infinitiv für ,an action bound to happen in a given counterfactual scenario“ siehe CGCG 34.17 (Hervorhebung hinzugefügt). 
Entlastung durch die Pragmatik etwa die Äußerung einer Absicht oft schon genügen, um direkt im Anschluss die Verwirklichung in der Erzählung über-

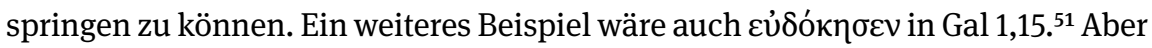
auch Gal 2,16-17 ist aufschlussreich.

\subsection{Beispiel Gal 2,16-17}

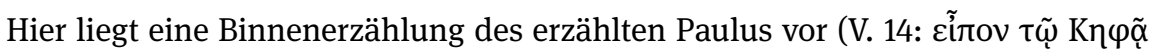

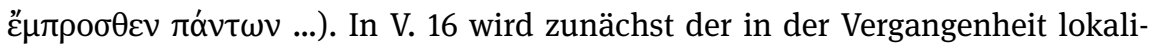

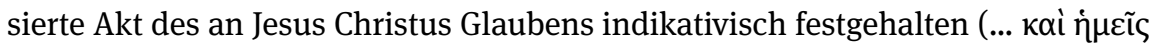

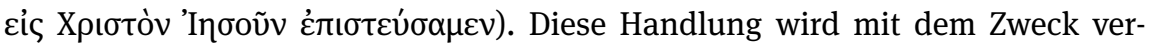
sehen: „... um aus Glauben Christi und nicht aus Werken des Gesetzes gerecht-

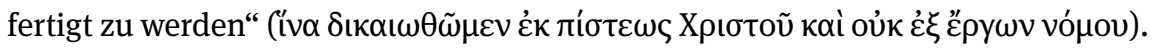
Der Akt des Glaubens wird mit Zitat aus Ps 142,2 begründet: „... weil aus Werken

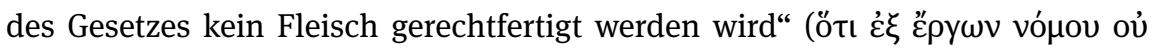

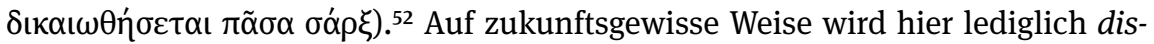
erzählt, nämlich jeglicher erfolgreiche Versuch einer Rechtfertigung auf der Grundlage von Gesetzeswerken. Über die demgegenüber aber tatsächliche, beab-

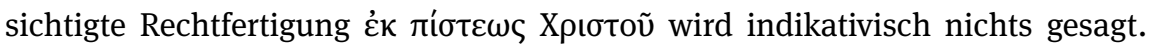
Lediglich zu Beginn des Verses ist diese prinzipielle Möglichkeit als „Wissen“ cha-

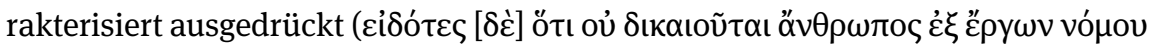

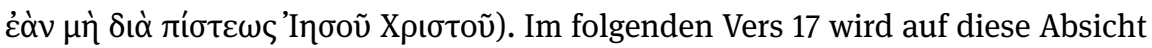
Bezug genommen: „danach strebend, in Christus gerechtfertigt zu werden“ ( $\varepsilon \dot{\imath} \delta \dot{\varepsilon}$

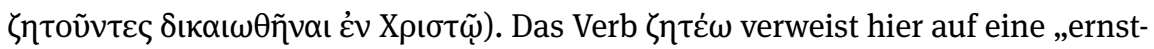
hafte Anstrengung zur Realisierung eines Wunsches, "53 wobei für letzteren das Verb des îv $\alpha$-Satzes explizit wiederholt wird. Das hier als aktives Bemühen vorgestellte Mittel zur Erlangung des Ziels ist der Akt des Glaubens aus dem Vorvers, der hier nicht mehr wiederholt wird, sondern schlicht durch die Charakterisie-

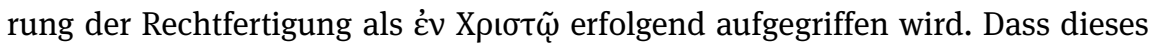
Bestreben erfolgreich war, dass der Glaube an Christus sich also als adäquates Mittel herausstellte, dass es zur Rechtfertigung in Christus kam, wird hier freilich nicht ausgesagt. Nichteinmal der Umstand, dass Paulus und Petrus hierdurch

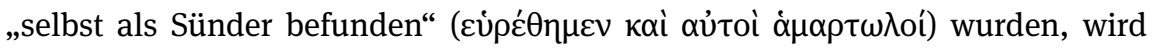
strenggenommen behauptet, denn es handelt sich hierbei um eine indefinite

51 Kapitel 5, Abschnitt 5. siehe oben, Kapitel 2.3.2 für Versuche, auch in Gal 1,16a eine weitere Ausgestaltung der Vorausdeutung auf die Mission zu sehen.

52 Zur Verneinung siehe GGNT 249a.

53 BDAG 3381,3: ,to devote serious effort to realize one's desire or objective. 


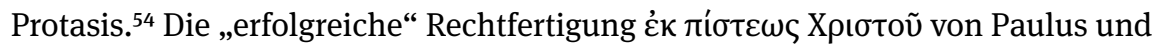
Petrus $^{55}$ im Vorfeld der Binnenerzählung ist vorausgesetzt und braucht in dieser nicht weiter erzählt zu werden.

\subsection{Beispiel Gal 2,2 und 4-5}

In anderen Fällen überbrücken Vorausdeutungen bei Paulus aber nicht nur in diesem Sinn Ellipsen in der Erzählung, sondern stellen durchaus anspruchsvollere Stilmittel der Leserlenkung dar. Zumindest an einem Beispiel soll im Folgenden noch der der heuristische Wert der Konzentration auf Vorausdeutungen auf die Vergangenheit (und Gegenwart) des Erzählers demonstriert werden. Es eignet sich hierfür Gal 2,4-5 besonders gut. Denn dass wir uns hier innerhalb einer Erzählung befinden, wird selbst von Kritikern des narrativen Ansatzes eingeräumt. ${ }^{56}$

Die geschilderte Episode des Besuchs in Jerusalem ist deutlich aus der Per-

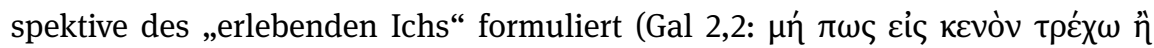

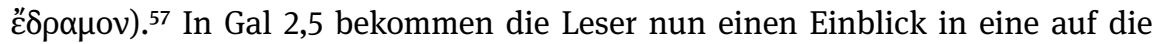
Zukunft der Erzählung ausgerichtete Prognose von Paulus und seinen Mitarbeitern: Sie widerstanden den „falschen Brüdern“ aus V. 4, „damit die Wahrheit des

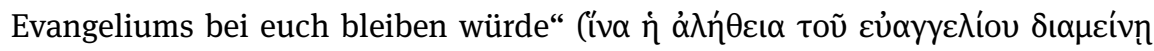
$\pi \rho o ̀ \varsigma$ ư $\tilde{\alpha} \varsigma)$.

Es liegt hier in V. 2 das vorausdeutende Motiv der Angst (Lämmert) beziehungsweise eine passive Prognose (Ryan), die zur einer zukunftsgestaltenden Handlung führt, deren Erfolg an dieser Stelle jedoch noch nicht festgehalten wird, vor. Die Galater werden auf diese Weise angehalten, der Figur des Paulus in Jerusalem ein Protonarrativ zuzuschreiben, von dem sie erkennen sollen, dass sein Plot dann auch dem tatsächlichen Verlauf der Dinge entspricht - ganz anders das Protonarrativ der „falschen Brüder,“ welches in 2,4 ebenfalls durch

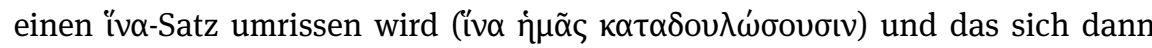
aber aufgrund der Initiative der Säulen in 2,6 als lediglich „virtuell“ herausstellt.

Auch für diese Passage Gal 2,4-5 gilt, dass nicht einfach nur Protonarrative von Erzählfiguren kommuniziert werden sollen (vgl. oben, Kapitel 10,

54 Vgl. unten, Kapitel 14, Abschnitt 5.2.1.

55 Siehe Heilig, „New Perspective“ und „Petrus-Tradition“ für eine Interpretation der Rede des Paulus mit einem Schwerpunkt auf der Betonung der gemeinsamen Erfahrungen der beiden Apostel.

56 Watson, „Story,“ 232.

57 Zur Differenz zwischen erlebendem und erzählendem Ich siehe Köppe und Kindt, Erzähltheorie, 221. 
Abschnitt 3). Vielmehr dienen auch sie der Leserlenkung und fordern dazu auf, die zu diesem Zeitpunkt der Erzählung in den Fokus gerückten möglichen Handlungsverläufe $\mathrm{zu}$ bewerten.

Unterstützt werden die Leser dabei durch spezifische zukunftsgewisse Vor-

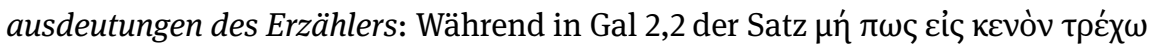

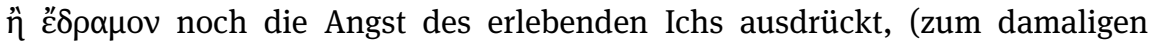
Zeitpunkt) umsonst zu laufen oder gelaufen $\mathrm{zu}$ sein, kommen in 2,4-5 bereits Hinweise auf den tatsächlichen Ausgang: Die falschen Brüder haben das Ziel,

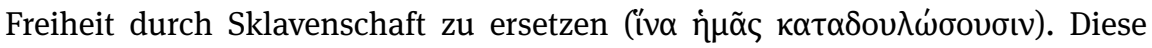

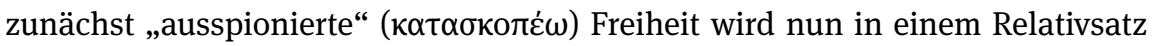

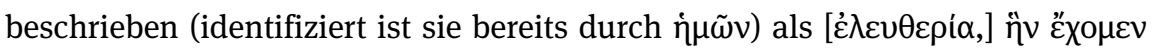

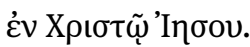

Durch die Wahl des Indikativ Präsens für $\chi \chi \omega$ wird eine auffällige Prolepse

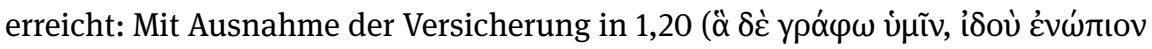

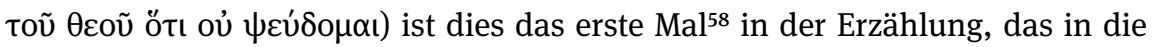
Gegenwart des erzählenden Ichs gesprungen wird. Durch die vermutlich vorliegende inklusive Verwendung des Personalpronomens ${ }^{59}$ wird hier zudem bereits eine recht spezifische zukunftsgewisse Vorausdeutung vorgenommen, nämlich auf die bis in die Gegenwart des Schreibens reichende Endsituation, ${ }^{60}$ welche auch die Galater mit einschließt - oder zumindest noch einschließen sollte, sofern sie nicht gerade im Begriff sind, eben jene Freiheit in Christus zu verwerfen.

Noch deutlicher wird dieser konkrete Bezug zur Situation der Galater dann freilich im Folgevers, in welchem vom Bleiben der Wahrheit des Evangeliums bei „euch“ die Rede ist. Genau genommen handelt es sich hierbei nur um eine zukunftsungewisse Vorausdeutung, denn der Finalsatz ist in seinem Bezug zur Wirklichkeit natürlich unbestimmt. Aber auch wenn natürlich noch nach dem Aufenthalt in Jerusalem Ereignisse denkbar sind, welche die Absicht hätten vereiteln können, so bestätigt zumindest der Fortgang der Erzählung ab V. 6, dass zumindest unmittelbar das für die Verwirklichung der Absicht Notwendige geschah. Nimmt man an, dass „die Freiheit, die wir in Jesus Christus haben“ aus V. 5 und „die Wahrheit des Evangeliums“ aus V. 6 zumindest teilweise referenzidentisch sind, dann weist die Rede vom präsentischen „Haben“ zudem nach, dass sich das beabsichtigte „Bleiben“ auch realisierte.

Dass mit $\delta \iota \alpha \mu \varepsilon v \omega$ auf einen Sachverhalt Bezug genommen wird, der von Jerusalem aus betrachtet noch in die Zukunft weist, ist offensichtlich. Auch wird

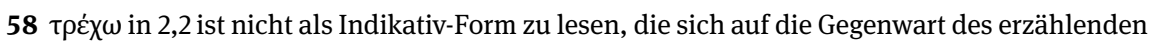
Ichs beziehen würde.

59 Wallace, Grammar, 397-399.

60 Siehe dazu oben, Abschnitt 3.1. 
für die Erstleser das mit der finalen Konnexion konkret Gemeinte insgesamt problemlos zu verstehen gewesen sein. Für die Analyse stellt die Vorausdeutung jedoch ein recht komplexes Problem dar, auch wenn Kommentare nicht selten ohne viel Aufhebens über sie hinweggehen. Letztlich geht es um die Frage, ob hier eine Vorausdeutung auf das Fortbestehen der Evangeliumsbotschaft auf Seiten des Paulus bis zu dessen Verkündigung in Galatien vorliegt oder ob der Fokus vielmehr auf dem die Galater betreffenden Handlungsstrang liegt und es darum geht, dass die bei ihnen lokalisierte Wahrheit des Evangeliums unbeschadet fortbestehen werde.

Eine der von BDAG angegebenen Bedeutungen für $\delta\llcorner\alpha \mu \varepsilon ́ v \omega$ lautet ,to continue in association with someone. ${ }^{61}$ Louw-Nida definieren ganz ähnlich: ,remain in an association for a period of time. ${ }^{62}$ Das Verb würde dann also das Anhalten eines bereits bestehenden Zustandes zum Ausdruck bringen. Eine solche Verbsemantik lässt sich im Rahmen der Annahme einer südgalatischen Adressatenschaft problemlos integrieren. ${ }^{63}$ Paulus würde dann auf eine zum Zeitpunkt des Besuchs in Jerusalem bereits erfolgt habende Missionstätigkeit in Galatien (während der „ersten Missionsreise“) zurückblicken. Sein Eintreten in Jerusalem für das von ihm verkündigte Evangelium hätte den Zweck gehabt, dafür zu sorgen, dass das bereits in Galatien Erreichte nicht zunichte gemacht würde. Im Kontext fände dies in V. 2

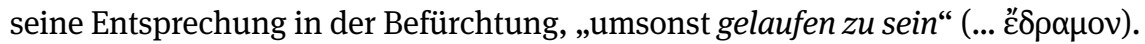

Narratologisch auffällig ist dann vor allem, dass Paulus direkt auf die Rezipienten des Briefes Bezug nimmt. Denn es besteht natürlich eine Kontinuität zwischen dem „Ihr,“ für welches Paulus sich in Jerusalem einsetzte und der

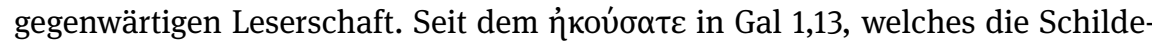
rung des Lebenswandels des frühen Paulus „im Judentum“ und damit die Erzählung als Ganzes einleitete, war diese in den Hintergrund geraten. Ihre direkte Anrede ist daher in Bezug auf die Fokalisierungsinstanz des erlebenden Ichs eine auffällige Metalepse:64 Der erzählte Paulus mag an „die Galater“ gedacht haben, der erzählende Paulus denkt an „euch.“

Wäre das Verb mit $\mu \varepsilon \tau \alpha \dot{~ k o n s t r u i e r t ~ w i e ~ i n ~ L k ~ 22,28 ~(u n d ~ a u c h ~ s o n s t ~ i n ~ v i e l e n ~}$ antiken Quellen), so müsste in der Exegese vermutlich völlig unstrittig sein, dass von einem anhaltenden „Bleiben“ die Rede is ${ }^{65}$ - und der Brief somit an den

61 BDAG 1880,3.

62 LN 34.3.

63 Siehe etwa Zahn, Galater, 96.

64 Siehe dazu oben, Kapitel 3, Abschnitt 5.2 zu den Erzählebenen und Kapitel 8, Abschnitt 5.4.2.5 ausführlich zu Gal 2,11-21.

65 Alternativ könnte man höchstens noch die 2. Person Plural als „euch (Heiden)“ meinend verstehen. Diese Option hat sich aber zurecht in der Exegese nicht durchsetzen können. 
südlichen Teil der Provinz Galatien adressiert war. Die Konstruktion mit $\pi \rho o ́ \varsigma$ lässt jedoch einen gewissen Diskussionsspielraum. Im NT ist sie sonst nicht

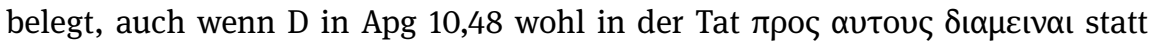
$\varepsilon \pi \iota \mu \varepsilon เ v \alpha \iota$ bietet.

Grundsätzlich ist ein Verständnis von einem Bleiben „bei“ jemandem natürlich durchaus mit dem Gebrauch der Präposition zu vereinbaren, wie etwa die Kombination mit $\dot{\pi} \pi \mu \varepsilon \dot{v} \omega$ in Gal 1,18 (vgl. auch 1. Kor 16,7) zeigt. Eine kurze

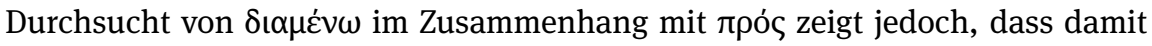
meist eine temporale Beziehung angezeigt wird. So notiert Theophrastos (De causis plantarum 4.1.10) das Erstaunen über manche Samen, welche trotz aller

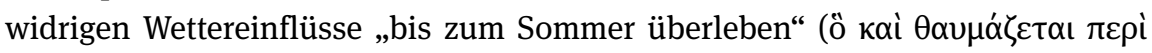

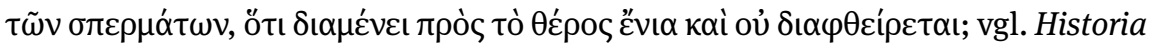

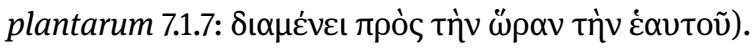

Analog muss man die Semantik von $\delta ı \alpha \mu \varepsilon v \omega$ auch in Gal 2,5 als ein ,anhaltendes Existieren' ausdrückend annehmen, ${ }^{66}$ wenn man von einer nordgalati-

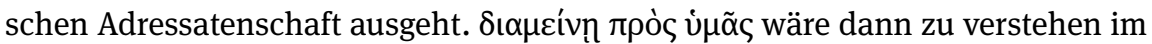
Sinn von einem „Erhaltenbleiben bis zu euch,“ also bis die Verkündigung dann später auch in der Landschaft Galatien stattfinden sollte.

Entsprechend übersetzt die NGÜ: „... denn die Wahrheit, die uns mit dem Evangelium gegeben ist, sollte euch unter allen Umständen erhalten bleiben.“ Diese Umgestaltung in eine kausale Konnexion nimmt dem Text freilich sein außerordentliches Gepräge. In dieser Übersetzung hat man den Eindruck, der Erzähler kommentiere aus seiner gegenwärtigen Position ein vergangenes Geschehen. ${ }^{67}$ Im Griechischen würde die Perspektive des erzählten Ichs aber viel unmittelbarer durchbrochen: Eine Handlung in der erzählten Vergangenheit wäre dann durch einen Zweck motiviert, welcher auf Figuren aus der noch ausstehenden erzählten Zukunft Bezug nähme!68

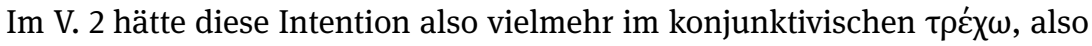
im noch nicht zum Abschluss gekommenen „Laufen,“ seine Entsprechung. Und so wie im Aristeasbrief (310) im Hinblick auf die LXX gesagt wird, sie solle wie

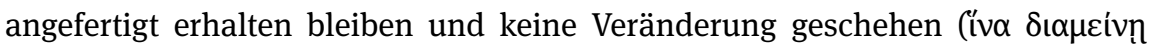

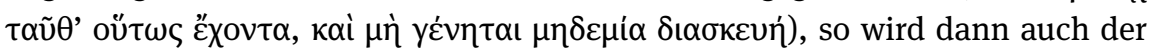

66 BDAG 1880,3. Ebenso LN 13.89.

67 Vgl. Duden 820.

68 Siehe etwa Betz, Galaterbrief, 177: „Paulus argumentiert, daß die ,Wahrheit des Evangeliums“ preisgegeben worden wäre, wenn er in Jerusalem nachgegeben hätte, und daß die Galater dann überhaupt kein Evangelium erhalten hätten.“ Mußner, Galaterbrief, 111: Das Verb sei „überzeitlich“ gemeint: „ein für allemal sollte die Wahrheit des Evangeliums erhalten bleiben und jetzt auch ,bei euch", den Galatern.“ 


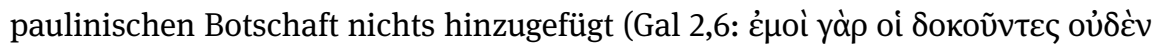

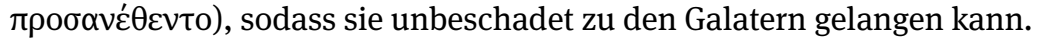

Diese Streitfrage kann an dieser Stelle nicht abschließend geklärt werden, auch wenn eine genauere Untersuchung schon aufgrund der Auswirkungen auf die Einleitungsfragen aber auch im Hinblick auf das paulinische Erzählen sehr angezeigt wäre. Zugunsten der erstgenannten Auslegung muss auf jeden Fall fest-

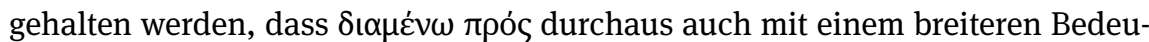
tungsspektrum der Präposition belegt ist. ${ }^{69}$ Auch scheint der ja völlig unstrittige

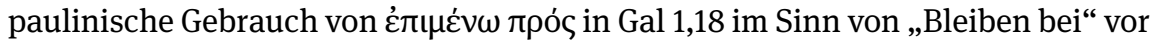
dem Hintergrund einer oberflächlichen Durchsicht des TLG ebenfalls recht außergewöhnlich, sodass das Argument fehlender Parallelen nur bedingt überzeugend ist. Auch darf nicht vergessen werden, dass $\delta \_\alpha \mu \varepsilon v \omega$ in der LXX durchaus häufig im Sinn eines bis zu einem bestimmten Zeitpunkt verlaufenden Bleibens oder Erhaltenbleibens gebraucht wird, dann aber gerade nicht $\pi \rho$ ó

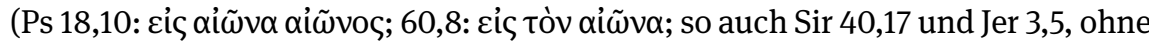

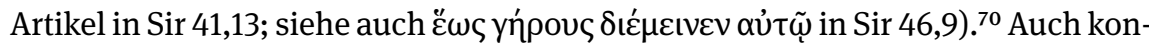
textuell ist die durch die nordgalatische Hypothese notwendig gemachte Interpretation recht problematisch. Denn in V. 4 ist die Intention der Falschbrüder ja

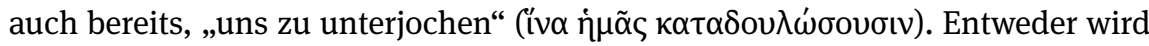
auch hier Erzählfiguren ein anachronistisches Motiv untergeschoben - oder man kann das Personalpronomen nicht inklusiv verstehen. Dann aber gilt dasselbe aber wohl auch für das in der 1. Person Plural formulierte Präsens-Verb im vorangehenden Relativsatz ( zumindest sehr unnatürlichen und sehr leicht missverständlichen Ausdrucksweise notwendig machen würde. ${ }^{71}$

69 Dion Chrysostomos, Or. 36.4: „einige - nicht viele - Türme bestehen, (allerdings) nicht gemäß

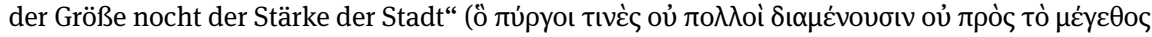

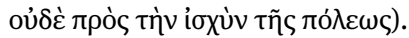

70 Das „Bleiben bei“ wird allerdings in Sir 12,15 und 44,11 (mit $\mu \varepsilon \tau$ ) $)$ und 22,23 (mit Dativ) ebenfalls alternativ ausgedrückt.

71 Für eine ausgeglichene Diskussion von Gal 2,4 siehe Coppins, Freedom, 93-103, der auch die überzeugenden Argumente für die Ursprünglichkeit von oĭ oủoć zusammenfasst. Problematisch scheint aber folgende Feststellung (S. 96): „Vv. 6-10 indicate that those of repute did not (attempt to) compel Titus to be circumcised." Es ist vor dem Hintergrund dieser Annahme, dass es in der Tat plausibler erscheint, die so generell klingende Aussage im Relativsatz in V. 4 sei nur auf Paulus und seine Mitarbeiter bezogen. Die Voraussetzung selbst ist jedoch durchaus problematisch, denn in den Versen 6-10 gibt es keinen Anhaltspunkt dafür, dass die Person des Titus überhaupt noch eine Rolle spielt. Zwar ist nicht klar, welche Handlung von den Falschbrüdern ausging, doch bereits die Formulierung in V. 3, dass „nicht einmal“ (oú8ć; vgl. BDAG 5423,3) Titus - der direkt in die Gemeinschaft der Jerusalemer Judenchristen Eingeführte (vgl. Kapitel 8, Abschnitt 5.2.4.2 
In jedem Fall weist der Ǐvo-Satz in Gal 2,5 aber über den zeitlichen Rahm der erzählten Situation in Jerusalem hinaus. Die Vorausdeutung ist alles andere als subtil. Dass sie dennoch bisher in der Galaterbrief-Auslegung kaum eine Rolle spielt, liegt wohl nicht zuletzt am Fehlen einer konsequent narratologischen Herangehensweise.

Das Versäumnis dieser Beobachtungen hat große Auswirkungen auf die Lektüre des gesamten Briefes. Nimmt man wahr, dass die in Gal 1,13 einsetzende Erzählung nicht einfach eine isoliert erfolgende Autobiographie darstellt, sondern dass sie durch bewusste Erzählzüge mit der konkreten Situation verwoben ist, so müssen zwangsläufig auch die über den ganzen Brief zerstreuten fragmentarischen Anmerkungen über die zu verschiedenen Zeitpunkten in Galatien geschehenen Ereignisse mit dieser Erzählung in Verbindung gebracht werden.

Diese umfassende Protoerzählung über die Interaktion von Paulus und den Galatern ${ }^{72}$ wird im Brief nicht vollständig entfaltet. Lediglich der „Prolog“ (unter der Annahme der nordgalatischen Hypothese) oder „Epilog“ (unter der Annahme einer südgalatischen Hypothese) auf dem Paulus betreffenden Handlungsstrang wird ausführlich narrativ vertextet (inklusive mehrerer in die Rahmenerzählung integrierter, Erzählfiguren zugeschriebenen, Binnenerzählungen).

Aber auch beispielsweise die Frage in Gal 3,3 - ,wollt ihr nun im Fleisch voll-

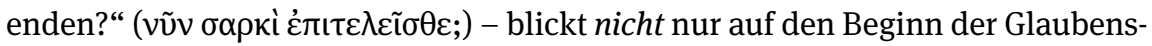

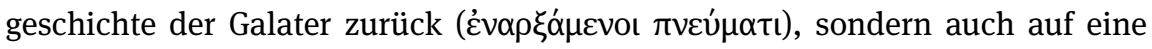
vermeintliche Endsituation, die so zwar nie erzählt aber zukunftsgewiss angedeutet war. ${ }^{73}$ Während die Exegese in Gal 3,1-4 zuverlässig den Kontrast zur Anfangszeit der Gemeinden herausgearbeitet hat und auch den Gegenwartsbezug im

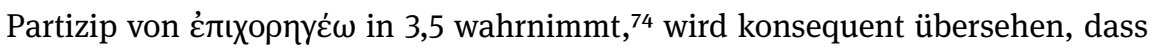
die Vorausdeutung in 2,4 und 2,5 einen Endzustand impliziert, der als eben so deutliche Kontrastfolie fungiert. Gerade darin liegt natürlich auch die in Gal 1,6 nicht näher erläuterte Ursache der Verwunderung des Paulus - dass nämlich das momentane Verhalten der Christen in Galatien dem rhetorisch vorausgesetzten „happy end“ zu widersprechen scheint.

Zusammenfassend lässt sich sagen: In Gal 2,4-5 begegnen uns einerseits zukunftsungewisse Vorausdeutungen sowohl des Paulus und seiner Mitarbeiter

zur eigentlich unmissverständlichen Wortstellung) - zur Beschneidung genötigt wurde und die generellen - die Heidenchristen in der Ferne betreffenden - Sorgen aus V. 2 sich somit als unbegründet herausstellten (vgl. einleitendes $\dot{\alpha} \lambda \lambda \alpha \dot{~ i n ~ V . ~ 3), ~ m a c h t ~ e s ~ b e r e i t s ~ v i e l ~ w a h r s c h e i n l i c h e r, ~}$ dass dieses Thema bereits mit V. 3 hinreichend besprochen wurde.

72 Siehe v. a. oben, Kapitel 3, Abschnitt 5.2 und Kapitel 8, Abschnitt 5.2.

73 Siehe dazu oben, Abschnitt 3.1.

74 Z. B. Fee, Galatians, 111. 
als auch der „falschen Brüder.“ Das Protonarrativ der letzteren stellt sich mit dem Fortgang der Erzählung von 2,6 als lediglich „virtuell“ heraus. Auf Rezipientenseite löst das Gegenüber der sich widersprechenden Protonarrative einen Prozess der Reflexion der noch ausstehenden Handlung der Rahmenerzählung aus. Dabei werden die Leser auch durch die als zukunftsgewiss und bis in die erzählende Gegenwart reichend zu betrachtende Vorausdeutung des Erzählers im Relativsatz in V. 5 gelenkt. Der so implizierte Endzustand braucht dann gar nicht mehr erzählt zu werden. Er stellt aber den Hintergrund dar, vor welchem das nun von den Galatern eigenmächtig hinzugefügte „Schlusskapitel“ umso schockierender erscheint.

\section{7 Übersicht über die Optionen}

Zusammenfassend lässt sich also sagen: Liegt das Erzählte in der Vergangenheit des Erzählers, so kann er die Erwartungen der Leser lenken, indem er durch zukunftsungewisse und zukunftsgewisse Vorausdeutungen der Erzählfiguren oder durch als eigene Kommentare gekennzeichnete zukunftsgewisse Vorausdeutungen aus der erzählenden Perspektive Bausteine für die Konstruktion der noch ausstehenden Handlung liefert.

Im Fall zukunftsungewisser Vorausdeutungen stellen die so evozierten Protonarrative lediglich Möglichkeiten (innerhalb der jeweiligen Erzählebene!) dar, im Fall zukunftsgewisser Vorausdeutungen handelt es sich um Prolepsen im eigentlichen Sinne, d.h., es werden diejenigen Erzählungen ausgeschlossen, welche das vorweggenommene Ereignis nicht integrieren können. ${ }^{75}$

Liegen die Ereignisse, von denen die Rede ist, auch aus Sicht des Erzählers in der Zukunft, kann dieser auf jeden Fall entsprechend seiner eigenen Prognosen zukunftsungewisse Vorausdeutungen anstellen. Abhängig von seinen Überzeugungen bezüglich der Möglichkeit menschlicher Einsicht in die Zukunft können unter Umständen auch zukunftsgewisse Vorausdeutungen von ihm formuliert werden. In diesem Fall könnte man auch mit Genette von „prädiktiver Erzählung“ beziehungsweise „früherer Narration“ des Erzählers (d.h. nicht einer Erzählfigur) - also auf der Ebene der Rahmenerzählung sprechen.

Die folgenden Beispiele fiktionaler Alltagserzählungen in Abb. 56 illustrieren die verschiedenen Möglichkeiten:

75 Das Gesagte setzt einen zuverlässig erzählenden Erzähler voraus. Vgl. Kapitel 3, Abschnitt 5.4. 


\begin{tabular}{|c|c|c|c|}
\hline & & $\begin{array}{l}\text { Zukunftsungewisse } \\
\text { Vorausdeutung }\end{array}$ & $\begin{array}{l}\text { Zukunftsgewisse } \\
\text { Vorausdeutung }\end{array}$ \\
\hline \multirow[t]{2}{*}{$\begin{array}{l}\text { Bezug auf } \\
\text { Vergangenheit des } \\
\text { Erzählers (der } \\
\text { Rahmenerzählung) }\end{array}$} & $\begin{array}{l}\text { Äußerung einer } \\
\text { Erzählfigur (inkl. } \\
\text { des erzählten Ichs) }\end{array}$ & $\begin{array}{l}\text { „Vor } 20 \text { Jahren sagte mein } \\
\text { Vater zu mir: ,Mit dieser } \\
\text { Ausbildung wirst Du die } \\
\text { nächsten } 10 \text { Jahre wohl } \\
\text { keine Arbeit finden."“ }\end{array}$ & $\begin{array}{l}\text { „Vorgestern sagte } \\
\text { mir eine } \\
\text { Wahrsagerin } \\
\text { voraus, dass ich } \\
\text { innerhalb von } 24 \mathrm{~h} \\
\text { einen Autounfall } \\
\text { haben würde." }\end{array}$ \\
\hline & $\begin{array}{l}\text { Äußerung des } \\
\text { Erzählers (der } \\
\text { Rahmenerzählung) }\end{array}$ & $\begin{array}{l}\text { „Es wird sich noch zeigen, } \\
\text { ob mein kindliches Ich mit } \\
\text { diesen Annahmen über die } \\
\text { Jugendjahre recht behalten } \\
\text { würde." }\end{array}$ & $\begin{array}{l}\text { „Damals wusste } \\
\text { ich noch nicht, } \\
\text { dass ich schon } \\
\text { bald eines } \\
\text { Besseren belehrt } \\
\text { werden sollte. }{ }^{676}\end{array}$ \\
\hline \multirow[t]{2}{*}{$\begin{array}{l}\text { Bezug auf Zukunft } \\
\text { des Erzählers (der } \\
\text { Rahmenerzählung) }\end{array}$} & $\begin{array}{l}\text { Äußerung einer } \\
\text { Erzählfigur (inkl. } \\
\text { des erzählten Ichs) }\end{array}$ & $\begin{array}{l}\text { „Jetzt studiere ich Medizin. } \\
\text { Schon als Kind hatte ich } \\
\text { gesagt: ,Eines Tages möchte } \\
\text { ich meine eigene Arztpraxis } \\
\text { haben.““ }\end{array}$ & $\begin{array}{l}\text { „Gestern erfuhr } \\
\text { ich von einer } \\
\text { Wahrsagerin, dass } \\
\text { ich noch lange } \\
\text { leben werde.“ }\end{array}$ \\
\hline & $\begin{array}{l}\text { Äußerung des } \\
\text { Erzählers (der } \\
\text { Rahmenerzählung) }\end{array}$ & $\begin{array}{l}\text { „Als ich gestern nach Hause } \\
\text { kam, fand ich Deine } \\
\text { Geburtstagseinladung im } \\
\text { Briefkasten. Ich habe } \\
\text { nächsten Samstag viel zu } \\
\text { tun, will aber gerne } \\
\text { versuchen, am Abend } \\
\text { vorbeizuschauen.“ }\end{array}$ & $\begin{array}{l}\text { „Lass uns schnell } \\
\text { den Champagner } \\
\text { öffnen - in zwei } \\
\text { Minuten ist } \\
\text { Mitternacht!“ }\end{array}$ \\
\hline
\end{tabular}

Abb. 56: Beispiele zukunftsungewisse und -gewisse Vorausdeutungen auf verschiedenen Erzählebenen und mit unterschiedlicher Beziehung zur Orientierungszeit des Rahmenerzählers.

Auf die Vergangenheit des Erzählers bezogene Vorausdeutungen implizieren mentale eingebettete Narrative und sind daher durchaus auch relevant, wenn man nach Protoerzählungen in einem Text sucht. Das Beispiel 2. Thess 3,7b-8 demonstrierte dies bereits, ebenso die Diskussionen oben (Abschnitt 6) zu Gal 2,2.4-5 und 2,16-17. Hier soll dieser Aspekt jedoch nicht weiter vertieft werden, da es sich bei solchen Vorausdeutungen letztlich um ein Mittel handelt, mit

76 Vgl. Duden 782 zum „Schicksalsfutur“ im Deutschen. 
welchem ein Autor explizite Erzählungen supplementiert, also eine erzählte Welt weiter ausgestaltet und dadurch die Lesererwartung anregt. Es handelt sich dabei also letztlich um eine Technik des expliziten Erzählens. Vorausdeutungen eines Erzählers auf die eigene Zukunft können demgegenüber immer nur Teil einer impliziten Erzählung sein, eines Narrativs, das zum momentanen Zeitpunkt nur „potenziell“ ist und dessen Erzählbarkeit sich erst noch erweisen muss.

Wie oben bereits angedeutet, gibt es gute a priori Gründe, um davon auszugehen, dass die Kategorie der Vorausdeutungen des Erzählers auf seine eigene Zukunft (und zwar sowohl zukunftsungewisser als auch -gewisser Natur) für die Analyse der Paulusbriefe bedeutsam ist: Zum einen äußert Paulus immer wieder Pläne, die seine Interaktion mit den Gemeinden betreffen, zum anderen nimmt er immer wieder zu eschatologischen Ereignissen Stellung.

Besonders gut identifizierbar sind die mit solchen Zukunftsbezügen verbundenen Protonarrative naturgemäß dort, wo sie mit expliziten Erzählungen im unmittelbaren Kontext verbunden sind, wo also eine die Vergangenheit betreffende Ereignisfolge in die Zukunft fortgesetzt wird und so die tatsächliche Erzählung in eine (zumindest zum momentanen Zeitpunkt noch) potenzielle Erzählung übergeht.

Im Folgenden soll am Beispiel von Röm 15,18-32 illustriert werden, wie eine Weitung des Fokus über explizite narrative Vertextungen hinaus und die Mitberücksichtigung von Ereignisbezügen, die auch vom Standpunkt des Paulus aus noch futurisch sind, dabei hilft, den Diskurs zu erhellen.

\section{Exemplarische Analyse der Vorausdeutungen in Römer 15,18-32}

\subsection{Ereignisse im Text}

Auf den ersten Blick scheint die Behandlung des Abschnitts aus narratologischer Perspektive ${ }^{77}$ kaum gewinnbringend: Die Verbformen sind überwiegend nicht-indikativisch, ${ }^{78}$ es liegt also auf jeden Fall keine sonderlich durchgehende Implementation eines strikt-narrativen Vertextungsmusters vor (vgl. Kapitel 8,

77 Siehe Horn, „Selbstverständnis“ zum Abschnitt im Dialog mit unterschiedlichen in der Forschung vertretenen Ansichten dazu, wie Paulus sich hier präsentiert (z. B. Paulus als „Ausleger und Erfüller der Schrift“). Hier steht mehr die Frage im Zentrum, wie Paulus (als „Erzähler“) dabei vorgeht.

78 Das Verhältnis ist 36:25. Im Einzelnen: 6x Konjunktiv (V. 20, 24 [2x], 31 [2x], 32), 10x Infinitiv (V. 18, 19, 20, 22, 23, 24 [2x], 26, 27, 30), 10x Partizip (V. 20, 23 [2x], 24, 25, 28 [2x], 29, 30, 31). 
Abschnitt 2). Zudem stellen Vergangenheits-Tempora keine klare Mehrheit dar (1x Imperfekt: V. 22, 6x Aorist: V. 18, 20, 21, 26, 27 [2x]), sondern stehen in einem ausgeglichenen Verhältnis zu Gegenwarts- (5x Präsens: V. 24, 25, 27 [2x], 30; 3x Perfekt: V. 21 [2x], 29) und Zukunftsbezügen (5x Futur: V. 18, 21 [2x], 28, 29). Betrachtet man eine Zusammenstellung all dieser Verbformen, so scheint zudem keine klare temporale Progression von Vergangenheit, Gegenwart und Zukunft noch ein offensichtlicher Zusammenhang der Einzelereignisse erkennbar:

- Paulus „wird nicht wagen,“ (V. 18: oủ yò $\rho$ to $\lambda \mu \eta \dot{\sigma} \sigma \omega$...)

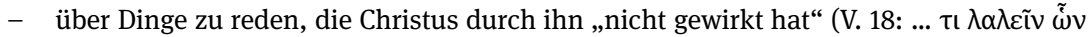

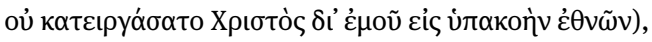

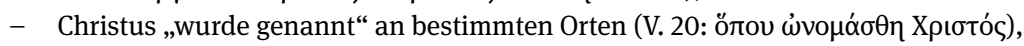

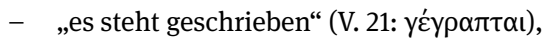

- innerhalb des Zitats in V. 21

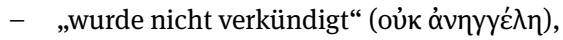

- „werden sie sehen“ (ő

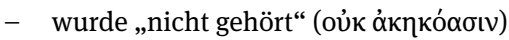

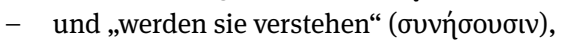

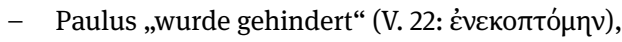

- er „hofft“ (V. 24: غ̇ $\lambda \pi i \zeta \omega)$,

- er „reist“ (V. 25: порєúo $\mu \alpha \mathrm{l})$,

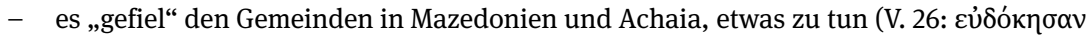

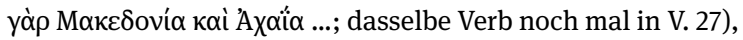

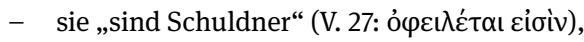

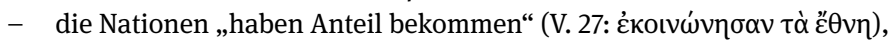

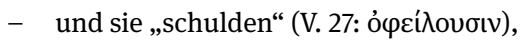

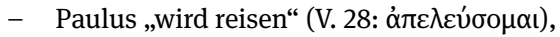

- er „weiß“(V. 29: oĩ $\delta \alpha)$,

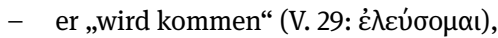

- er „bittet“ (V. 30: П $\alpha \rho \alpha \kappa \alpha \lambda \tilde{\omega})$.

Schränkt man die Suche weiter auf Formen der Vergangenheits-Tempora ein, so „erzählt“ Paulus lediglich in V. 22 in der 1. Sg. von Erlebtem (,autobiogra-

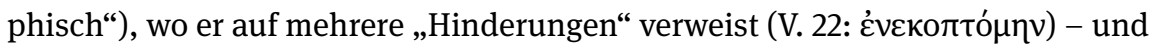
in V. 20 in der 3. Sg. von einer Sache, die er gerade nicht erlebt hat - nämlich die Missionierung von Menschen in Regionen, die er nicht besuchte (öтоv

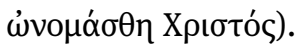

Der Text weist also gerade keine leicht identifizierbare explizite Erzählung vergangener Ereignisse auf, deren Plot man dann recht unkompliziert mit erwähnten zukünftigen Geschehnissen verbinden könnte. Doch gerade dies macht die Passage als Beispieltext sehr aussagekräftig - denn wie im Folgenden gezeigt werden soll, ergibt die sorgfältige Untersuchung der (Vergangenheit, Gegenwart und Zukunft betreffenden) Bezüge dennoch ein komplexes und zugleich kohärentes Protonarrativ. Folgt man diesem impliziten Handlungsverlauf (und seinen potenziellen Alternativen), so wirft dies neues Licht auf den Text, wodurch 
Struktur und Intention geklärt werden können. Dabei ist in der Analyse streng darauf zu achten, aus welcher Perspektive relative temporale Bezüge vorgenommen werden - also vor allem, ob im Text das erzählende Ich des Briefschreibers oder das erzählte Ich eines in der Vergangenheit anzusiedelnden Paulus den Fixpunkt der Betrachtung liefert.

\subsection{Der Rückblick des Erzählers V. 18-19}

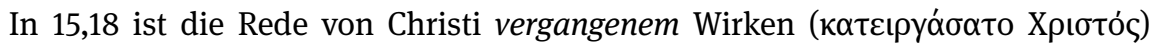

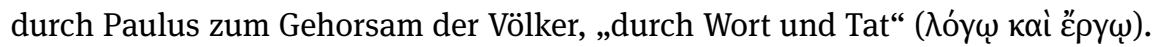
Vers 19a expliziert die göttlich wirksame Kraft und 19b beschreibt das mit $̋ ّ \tau \tau$ eingeleitete und durch die Wahl des Infinitiv Perfekt (siehe oben, Kapitel 6, Abschnitt 7.2.1 zur Konstruktion) unterstrichene Resultat: In der Gegenwart des Schreibers ist das Evangelium von Jerusalem aus bis nach Illyrien „vollendet.“

\subsection{Die Rückblende in V. 21-22}

Vers 20 ist, anders als das die meisten Übersetzungen nahelegen würden, ${ }^{79}$ eine modale Umstandsangabe ${ }^{80}$ und erläutert, dass Paulus in dieser - nun abgeschlossenen - Periode dem mit Jes 52,15 verbundenen Grundsatz (V. 21) folgte, Christus in missionarischem Neuland zu verkündigen. Das Zitat selbst wird als

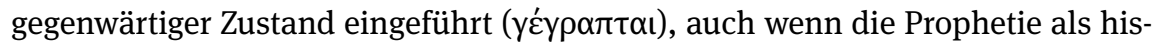
torische Äußerung natürlich in die Vergangenheit zurückführt. Der Fokus liegt jedoch nicht auf dieser „Vorgeschichte.“ Denn während Paulus etwa in Gal 3,8 die „Schrift“ durch den Indikativ Aorist in der erzählten Vergangenheit handeln lässt und durch das Futur die Verheißung als vom gegenwärtigen Standpunkt erfüllt darstellt, hat das Schriftzitat hier eine ganz andere Funktion: Es skizziert die Voraussetzungen der paulinischen Mission, wie sie ab 15,18 bereits beschrieben wurde. Als Begründung der Motivation des erzählten Ichs des Paulus nimmt es Vergangenheit, Gegenwart und Zukunft der erzählten Vergangenheit in den Blick, es handelt sich also um eine (zukunftsgewisse, da prophetische) Vorausdeutung auf die Vergangenheit des Paulus: In der (vom vergangenen Standpunkt aus

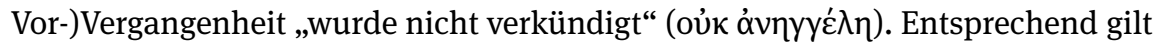

79 Etwa Luther mit Neuansatz: „So aber setze ich ...“

80 Siehe NSS 948. Weshalb es laut Wilk, Bedeutung, 81 aufgrund des vom Partizip abhängigen Infinitivs „nicht an[geht],“ die Syntax so aufzulösen, ist unklar. 
für die Gegenwart (des erzählten Ichs) dass der Apostel von Menschen umgeben ist, die „unerreicht“ sind (so könnte man das im Deutschen schwer zu fassende

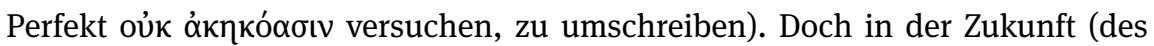
erzählten Ichs!), so verheißt Jes 52,15 dem berufenen Apostel, „,werden sie sehen“

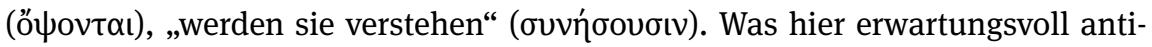
zipiert wird, ist bereits in 15,15-19 als tatsächlich eingetreten geschildert worden. Das Zitat in Röm 15,21 stellt streng genommen also eine Rückblende dar in ein mentales Narrativ des frisch berufenen Paulus, der die Verkündigung erst noch vor sich hat, ihren Erfolg aber bereits verheißen weiß.

Wilk ist daher durchaus zuzustimmen, wenn er hierin eine wichtige Bestimmung des paulinischen Selbstverständnisses sieht. ${ }^{81}$ Mit Vorsicht zu behandeln ist jedoch die Einschätzung, dass es sich hierbei um einen „,Basistext“ für den geplanten Rombesuch“ handle. ${ }^{82}$ Zweifellos richtig ist, dass die durch das Zitat identifizierten Heiden letztlich mit denen gleichzusetzen sind, „denen er das Evangelium gepredigt hat bzw. predigen will“"83 - also auch noch in der Zukunft des erzählenden Ichs. Hätte Paulus allerdings tatsächlich einen Schwerpunkt darauf legen wollen, durch das Zitat das Wesen „sein[es] Verhältnis[es] zur Gemeinde in Rom [zu] definieren, "84 dann wäre der Fortgang des Textes äußerst unpassend formuliert. Denn in V. 22 verknüpft Paulus seine bisherige Missionsarbeit dann explizit mit dem generellen Thema eines Besuchs bei den Römern,

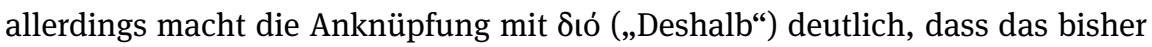
Erzählte gerade die Funktion hat, zu erklären, weshalb Paulus bisher nicht nach Rom gekommen ist. ${ }^{85}$

81 Wilk, Bedeutung, 400 und 402.

82 So Wilk, Bedeutung, 82. Hervorhebung nicht im Original. Vgl. Wilk, Bedeutung, 392: „Der hohe Stellenwert jener Verheißung ... liegt darin, daß Paulus mit ihr zugleich ... seine geplante Spanienmission legitimieren und sein Verhältnis zur Gemeinde in Rom definieren kann.“

83 Wilk, Bedeutung, 176. Hervorhebung nicht im Original.

84 Wilk, Bedeutung, 392.

85 Diese Anknüpfung durch den konsekutiven Konnektor hat in der Exegese für Verwirrung gesorgt: Der in V. 22 implizite Wunsch des Kommens nach Rom scheint sich schlecht als Folge der in V. 20-21 geäußerten Präferenz unmissionierter Gebiete zu ergeben. Die meisten Exegeten sehen daher einen weiteren Bezug der Resultat-Proposition zur „Aufgabe im Osten (VV 19ff)“ (Schlier, Römerbrief, 434). Paulus sei durch die (in V. 21 begründete) Tätigkeit schlicht zu beschäftigt gewesen. Der Ind. Perf. in V. 19 bringt jedoch wie gesagt den Abschluss dieser Tätigkeit zum Ausdruck, macht also eine Aussage über die gegenwärtige Situation und nicht direkt über die vorangehende Beschäftigung. Es liegt somit näher, dass der Konsekutivsatz hauptsächlich das ab V. 18 angeführte, auf den Gehorsam der Völker hinwirkende Agieren im Blick hat (so ist wohl auch Wilckens, Brief III, 124 zu verstehen), wie es Paulus für seine erzählte Vergangenheit vorhergesagt worden war. 
Das „Ereignisbündel,“86 das in V. 22 im mediopassiven (keine weitere Erzähl-

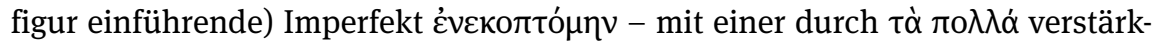
ten iterativen Nuancierung - zum Ausdruck kommt, ist also chronologisch auf die Zeitspanne bezogen, die durch die „Vollendung“ (V. 19) begrenzt wird. Das Zitat in V. 21 skizziert folglich einen Missionsplan, der mit diesem Zeitpunkt (vorerst) beendet ist (auch wenn - um Wilk indirekt recht zu geben - die nun für die Zukunft angestellten Pläne natürlich demselben „Handlungskriterium“ folgen). ${ }^{87}$

\subsection{Die Rückkehr zur simultanen Narration in V. 23-24a}

Dass dieser Zeitpunkt im Jetzt des Erzählers liegt, macht V. 23 durch vuví deutlich. In die Erzählung über seine bisherige Evangelisierungs-Tätigkeit waren genügend Vorausdeutungen enthalten, um nun an dieser Stelle eine Schilderung der noch zu verwirklichenden Pläne erwarten zu lassen. Und in der Tat scheinen die nochmals die gegenwärtige Situation umreißenden Partizipien in V. 23 die Ausgangssituation für eine solche Reise abzustecken: Der Verweis auf fehlenden

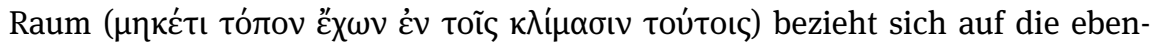
falls geographisch umrissene Vollendung in V. 19 und die Versicherung einer seit

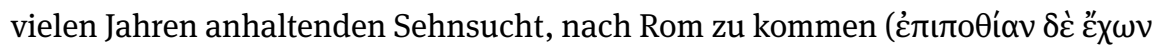

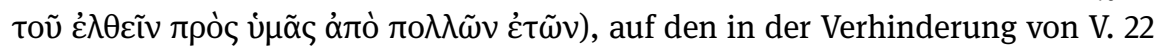
implizierten Wunsch eines solchen Besuches.

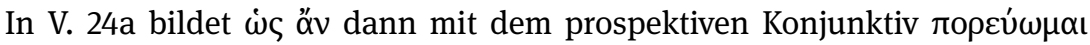
einen Temporalsatz, ${ }^{88}$ der am besten als Spezifizierung dieses zweiten Partizips zu sehen ist: ${ }^{89}$ Paulus hatte schon lange das Verlangen, zu „euch zu kommen ...

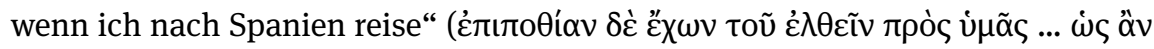

86 Vgl. Kapitel 3, Abschnitt 4.2.

87 Wilk, Bedeutung, 380.

88 GGNT 210i. Vgl. NSS 949.

89 Vgl. die Übersetzung von Menge: „23 Nun aber habe ich keine Aufgabe mehr in diesen Ländern, habe aber seit vielen Jahren das Verlangen, zu euch zu kommen, 24 wenn ich nach Spanien reisen werde.“ Alternativ muss der Temporalsatz auf den mit $\dot{\varepsilon} \lambda \tau i \zeta \omega$ eingeleiteten Satz bezogen

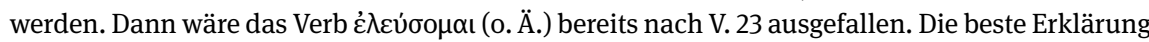
für die unvollständige Syntax ist jedoch, dass Paulus mit yó $\rho$ erst in V. 24 den begonnen Satz unterbricht. 


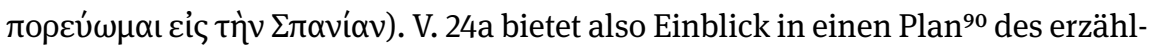
ten Ichs, den auch das erzählende Ich im Jetzt noch hegt. ${ }^{91}$

\subsection{Der Übergang zur prädiktiven Narration in V. 24b-25}

Diesen fühlt sich Paulus nun gezwungen, genauer zu erörtern, weshalb er den begonnen Satz abbricht ${ }^{92}$ und in V. 24b genauer darlegt, wie die Reise nach Spanien mit dem Besuch in Rom zusammenhängt: Die gegenwärtige Hoffnung

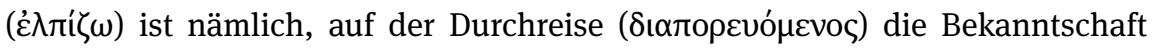

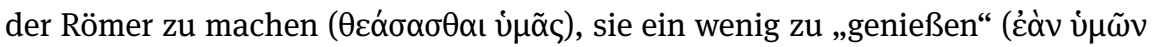

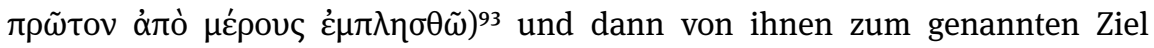

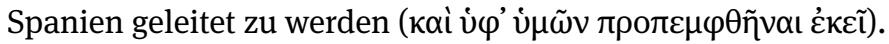

Dass Paulus dieses Vorhaben in V. 28 etwas redundant nochmal äußert ('́)

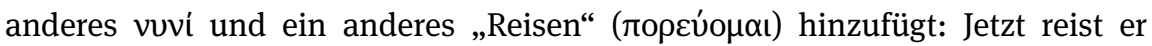
zunächst nach Jerusalem, um den Heiligen zu dienen.

\subsection{Die Rückblende V. 26-27 und Vereinigung der Handlungsstränge in V. 28a}

V. 26 bis 27 stellt dann eine Analepse dar, in welcher die bereits geschehene Kollekte erzählt wird. Die auffällige Zurücknahme seiner eigenen Person in dieser

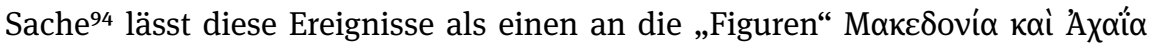
gebundenen Handlungsstrang erscheinen. ${ }^{95}$

$\mathrm{Zu}$ einer Verbindung der beiden Handlungsstränge kommt es in V. 28a, wo

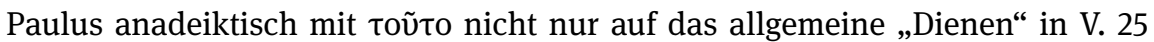
Bezug nimmt, sondern auch zum Ausdruck bringt, dass er die Bemühung der Hei-

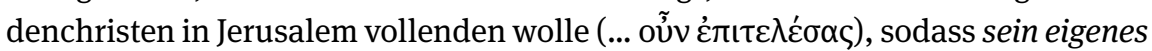

90 Zum Planen des Paulus in diesem Abschnitt insgesamt, siehe auch unten, Kapitel 14, Abschnitt 3.4.6.

91 Zur Überlagerung siehe oben, Kapitel 8, Abschnitt 7.4 im Kontext der „eingeschobenen Narration.“

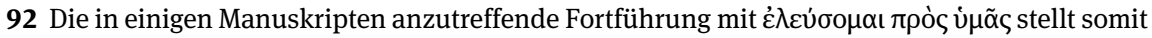
tatsächlich die plausibelste Auflösung der unvollständigen Syntax dar.

$93 \mathrm{Zu}$ ćóv vgl. unten, Kapitel 14, Abschnitt 5.2.2.2. Zum Adverb siehe bereits oben, Kapitel 7, Abschnitt 5.2.2.

94 Siehe etwa Schlier, Römerbrief, 436.

95 Zur Verknüpfung von Handlungssträngen mit einzelnen Erzählfiguren siehe oben, Kapitel 3, Abschnitt 5.3. 


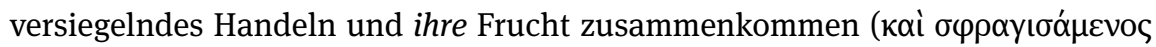

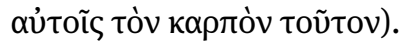

Vor diesem Hintergrund kann eigentlich keine Rede davon sein, dass Paulus in V. 25 durch die Erwähnung seiner Jerusalem betreffenden Pläne „überraschend auf eine andere Reise zu sprechen [kommt]." ${ }^{96}$ Vielmehr macht dieser Zug erzählerisch durchaus Sinn: Denn das von ihm in V. 24b so ausführlich dargestellte Protonarrativ einer Reise über Rom nach Spanien kann nur dann zur „Erfolgsgeschichte“ werden, wenn diese gegenwärtige Aufgabe erfolgreich gemeistert wird.

\subsection{Die Ankündigung des Besuches in V. 28b-29}

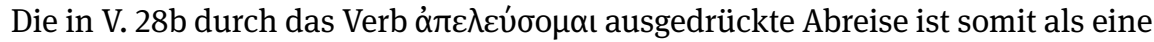
lediglich gewollte zu verstehen (modales Futur). ${ }^{97}$ Dieser Vorbehalt ist nun freilich in V. 29 fallengelassen, wenn Paulus versichert:

oĩo $\alpha \dot{\varepsilon}$

$$
\begin{aligned}
& \text { ötı } \rightarrow
\end{aligned}
$$

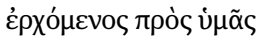

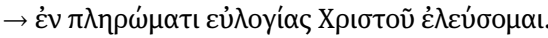

Die im einleitenden Verb zum Ausdruck kommende Gewissheit ${ }^{98}$ betrifft nicht nur die Art und Weise eines eventuellen Besuches. Vielmehr wird das Kommen selbst als zukünftig geschehend indikativisch postuliert, wobei das Partizip nur noch einen temporalen Umstand und nicht mehr eigentlich eine Bedingung zum Ausdruck bringt. ${ }^{99}$

\subsection{Die Römer als zentrale Figur der Protoerzählung in V. 30}

Dass Paulus direkt im Anschluss (V. 30) die Römer eindringlich zur Fürbitte ermahnt, scheint von manchen Exegeten als eine neuerliche Dämpfung des paulinischen Optimismus betrachtet zu werden. ${ }^{100}$ Die rhetorische Gestaltung ergibt aber durchaus Sinn, wenn man beachtet, wie Paulus das in diesem Abschnitt

96 Schlier, Römerbrief, 435.

97 Vgl. GGNT 202b zum voluntativen Sinn des Indikativ Futur. Siehe unten, Abschnitt Kapitel 14, Abschnitt 3.1 zur Abschwächung von Aussagesätzen.

98 Vgl. unten, Kapitel 13, Abschnitt 3 zu abhängigen Behauptungssätzen.

$99 \mathrm{Vgl}$. NSS 949.

100 Etwa Schlier, Römerbrief, 437: „Aber der Gedanke an Jerusalem läßt ihn noch nicht los.“ 
zugrundeliegende Protonarrativ bearbeitet: Sein schon lange feststehender (V. 22-23) Plan soll den Römern möglichst plastisch vor Augen gemalt werden, damit sie in die Lage versetzt werden, sich selbst innerhalb dieses noch potenziellen Narrativs zu verorten. Nachdem diese Orientierung in V. 29 ihre Klimax erreicht hat, ist genau der richtige Zeitpunkt, um das Mittel einzuführen, mit deren Hilfe dieser Punkt der Handlung erfolgreich erreicht werden soll.

\subsection{Das „Happy End“ in V. 31-32}

Die Römer werden also vom „prädiktiv erzählten“ Besuch des Paulus zurückgeführt in die Gegenwart, in welcher ihr Gebet dafür sorgen kann, ${ }^{101}$ dass in der nahen Zukunft Paulus' Besuch in Jerusalem erfolgreich verlaufen wird (V. 31), ${ }^{102}$

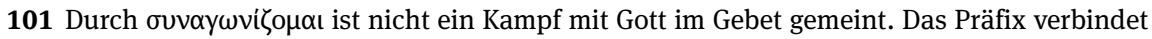
vielmehr die Handlungen von Paulus auf der einen Seite und die der Römer auf der anderen. Durch das Gebet partizipieren sie direkt an den Bemühungen des Paulus, die vor ihm liegenden Schwierigkeiten zu überwinden. Vgl. Poplutz, Athlet, 384-391.

102 Das ìva in V. 31 leitet einen Begehrsatz ein (vgl. GGNT 272b). Das genaue Verhältnis der durch die beiden Konjunktive ausgedrückten Ereignisse ist eine strittige Frage, die hier nicht diskutiert werden kann, da sie mit weitreichenden Vorentscheidungen zum Verhältnis des Paulus zur Jerusalemer Gemeinde zusammenhängt. Vgl. etwa Wilckens, Brief III, 128-129: „Zwar könnte die Abfolge von V31a.b durchaus darauf schließen lassen, daß zwischen der Gefahr jüdischer Verfolgung und der der Nichtannahme der Kollekte seitens der Urgemeinde ein ursächlicher Zusammenhang besteht. Doch der kann dann nur darin liegen, daß ein Teil der Jerusalemer Christen im Haß gegen Paulus mit den Juden in Judäa zusammensteht.“ White und Batson, „Paul's Collection“ haben kürzlich dafür argumentiert, dass die singuläre Variante in $\mathrm{P}^{46} \Delta \mathrm{IAT} \Omega N A \Gamma I \Omega N$ mehr Beachtung verdient. Sie meinen (S. 110), dass drei Interpretationen möglich seien: (1) Paulus bittet um Gebet, dass die Sammlung den Christen in Jerusalem (den „Heiligen“; Röm 15,25) gefällig sein möge, wobei die Übergabe durch die Gesandten seiner Gemeinden (ebenfalls „Heilige“) erfolge. (2) Paulus behalte priesterliche Terminologie bei und hoffe, die Sammlung, die durch die Gesandten seiner Gemeinden Gott als Opfer entgegengebracht werde, möge diesem wohlgefällig sein (vgl. Röm 15,16). (3) Die Heiligen sind die Christen in Judäa, durch welche nun die Sammlung an alle Armen in Jerusalem - ob gläubig oder ungläubig - erfolgen soll. Eine umfassendere Untersuchung relevanter Belege scheint angezeigt, insbesondere zur Evaluie-

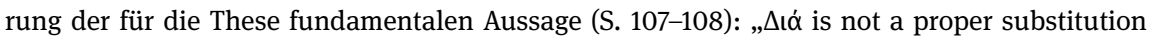
for the dative." In der Tat übernehmen Dativ- und Präpositionalkonstruktion im Normalfall unterschiedliche Funktionen, wenn die „Wohlgefälligkeit“ einer Sache ausgesagt wird (vgl. 1. Petr

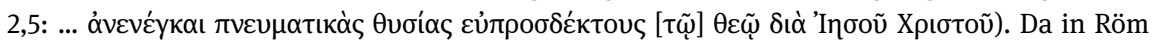
15,31 jedoch unter Annahme des rekonstruierten Textes ursprünglich keine Instrumentalangabe gestanden hätte, scheint es leichter vorstellbar, dass der Dativ instrumental aufgefasst wurde, da

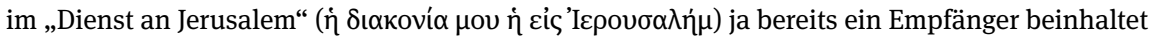
gewesen wäre. 
damit $^{103}$ (V. 32) Paulus letztlich auch in die Situation versetzt wird, sich bei den

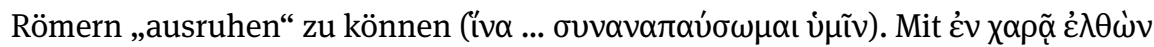
$\pi \rho o ̀ \varsigma$ ن̀ $\mu \tilde{\alpha} \varsigma$ in V. 32 ist vermutlich nicht modal auf dieses Ausruhen Bezug genommen, ${ }^{104}$ sondern die zeitlich vorausgehende Ankunft noch ein weiteres Mal aus-

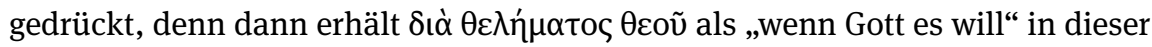
Partizipialkonstruktion eine angemessene Funktion. ${ }^{105}$ Die temporal noch weiter in die Zukunft weisende Handlung des von Paulus gehegten - und nun von den Römern hoffentlich geteilten - Planes braucht nicht mehr thematisiert zu werden, da sie durch den Einschub in 24b bereits proleptisch zur Sprache kam. Mit dem

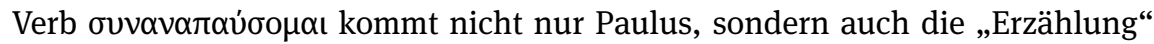
zur Ruhe.

Die Ergebnisse dieser schlaglichtartigen Beleuchtung der narrativen Grundstruktur dieses Abschnitts können wie folgt zusammengefasst werden: Paulus erzählt vergangene Geschehnisse und kreiert dabei durch Vorausdeutungen eine Erwartungshaltung für eine Schilderung des Jetzt-Zustands. Er erreicht das unter anderem durch die Integration mentale Ereignisse wie Wünsche und Pläne in seine explizite Erzählung. Das dadurch implizite Protonarrativ verknüpft er sowohl mit seiner späteren, zum Zeitpunkt des Schreibens gegenwärtigen, Situation als auch mit der noch späteren, auch für das erzählende Ich noch ausstehenden, Zukunft.

Es entsteht auf diese Weise ein komplexes Netz an Ereignisbezügen, welches sich jedoch $\mathrm{zu}$ einem kohärenten Handlungsstrang mit mehreren möglichen Abzweigungen zusammenfügt. Dieser wird aufwendig und nach verschiedenen Seiten abgestützt, etwa durch eine Rückblende (vgl. V. 27) und sogar durch argumentative Vertextungen innerhalb dieser Analepse. Alternative Handlungsstränge (etwa durch das Motiv der Angst evoziert), sowie eine plastische Ausgestaltung der noch zukünftigen und damit unsicheren Ereignisse dienen dazu, den noch ausstehenden Handlungsverlauf den Lesern nahezulegen. Paulus' Protonarrativ soll auch das ihrige werden.

Es wäre daher verfehlt, die Kommunikationsintention (siehe Kapitel 8, Abschnitt 4) des Paulus als lediglich informierend-feststellend zu klassifizieren, als ginge es ihm nur darum, seinen Besuch - quasi der Höflichkeit halber - ,anzukündigen." Es wäre ebenso noch immer zu kurz gegriffen, würde man die Intention des Kommunikationsversuches in der Übernahme einer bestimmten Bewertung des Besuches sehen. Vielmehr repräsentiert die Passage überhaupt nicht den

103 ivo in V. 32 bringt die finale Konnexion zum Ausdruck (GGNT 336a).

104 Erwogen etwa von NSS 950.

105 Die Variante mit Konjunktiv und koordinierender Konjunktion (

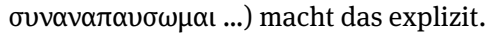


Intentionstyp „Glauben“ - sondern vielmehr „Wollen.“106 Denn berücksichtigt man die Kategorie der Protoerzählung des Paulus in diesem Text und bedenkt man die entscheidende Rolle, welcher in dieser dem Ereignis(bündel) des römischen Gebetes zukommt, wird deutlich, dass das Hauptanliegen ${ }^{107}$ des Paulus die Moti-

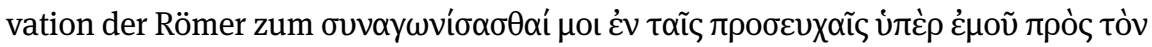
$\theta \varepsilon o ́ v$ (V. 30) ist. Diese starke Anbindung des Erfolgs in Jerusalem an die Unterstützung durch die Römer im Gebet stellt sicherlich eine erstaunliche Verknüpfung an Erzählsträngen dar, ist aber für Paulus durchaus nicht ungewöhnlich. ${ }^{108}$

\subsection{Ein impliziter Epilog?}

Zudem darf nicht übersehen werden, dass Paulus durch die erhoffte Einbindung der Römer in sein Protonarrativ als ihm im Gebet Beiseitestehende noch einen weiteren Effekt erzielt: Wenn sie sich tatsächlich entscheiden sollten, in der nahen Zukunft in der Fürbitte zu partizipieren, übernehmen sie dadurch einen Plan, in welchem sie auch auf ganz praktische Art und Weise als Hilfsfiguren für die Implementation des von Paulus anvisierten Endzustandes vorgesehen sind.

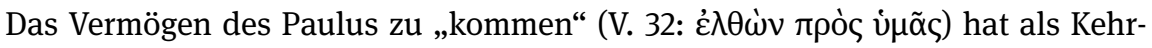
seite schließlich auch die Bereitschaft, ihn zu empfangen - und die darin zum Ausdruck kommende Bereitwilligkeit zur Unterstützung ist, wie Paulus nur kurz

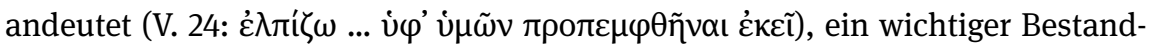
teil seines Planes. Die in Röm 15,18-32 zum Ausdruck kommenden Protonarrative motivieren daher nicht nur zum Gebet, sondern indirekt auch zu einer noch weiter in der Zukunft liegenden Unterstützung.

\subsection{Fazit}

Wie folglich die Erörterung dieses Abschnitts gezeigt hat, führt die Konzentration auf Ereignisse als Grundbausteine von Erzählungen interessanterweise hier gerade nicht dazu, dass die narrative Vertextung als primär informierende Strategie in den Fokus gerückt und die Aufforderung zum Gebet und Geleit als

106 Vgl. zu dieser Thematik der Textfunktion oben, Kapitel 8, Abschnitt 4.

107 Wilckens, Brief III, 128 erkennt richtig, dass es sich hierbei um „das Wichtigste“ handelt und identifiziert die relevanten Signale im Text korrekt. Es fehlt jedoch die auf dieser Grundlage zu erfolgende Bewertung der Textstruktur als Ganzer.

108 Teilweise ohne genaue Zweckangabe (1. Thess 5,25). In den umstrittenen Paulusbriefen vgl. Kol 4,3 und 2. Thess 3,1. 
subsidiäre Sprechhandlung betrachtet würde. ${ }^{109}$ Vielmehr wird gerade durch die Beachtung der grundsätzlich narrativen Struktur deutlich, dass die narrativen Elemente eine stützende Funktion für die auf das Handeln der Römer ausgerichtete Aufforderungen (die direkte in V. 30 und die indirekte in V. 24) hat.

Wird die von Paulus dem Abschnitt zugrunde gelegte narrative Struktur nicht wahrgenommen, so bleibt auch die Wahrnehmung der Kommunikationsintention, also die Funktion des Abschnitts, unpräzise. ${ }^{110}$ Damit korreliert dann der Eindruck einer fehlenden Kohärenz in der Struktur des Diskurses (etwa im Hinblick auf den „eingeschobenen“ Reiseplan in V. 25) - ein Eindruck, der sich bei Betrachtung der narrativen Tiefenstruktur als nur sehr oberflächlich zutreffend herausstellt.

Diskutiert werden kann natürlich, ob Paulus den hier skizzierten Plot tatsächlich mental als verbale Erzählung, als vollständig ausgebildete Protoerzählung, simuliert hat, ob er sich also in Gedanken auf einen zukünftigen Standpunkt in Spanien gestellt hat und aus dieser Perspektive rückblickend zusammenfasste, wie er dieses Ziel zu diesem Zeitpunkt erreicht haben würde. Unstrittig scheint jedoch, dass Paulus eine recht weit fortgeschrittene Narrativisierung vorgenommen hat (vgl. Kapitel 9, Abschnitt 4): Aus der potenziellen Zukunft wurden sehr spezifisch Ereignisse von ihm selektiert und in eine temporale und sinnhafte Ordnung untereinander und mit Ereignissen der Vergangenheit und Gegenwart gebracht. Das so gebildete Netz an Ereignissen, mit seinen verschiedenen, an unterschiedliche Figuren gebundenen Handlungssträngen, den gewünschten und unerwünschten Weiterentwicklungen und den als Schlüsselhandlungen identifizierten potenziellen Geschehnissen ist Paulus bereits im Abfassungsakt mental präsent, sodass er seinen rhetorischen Absichten entsprechend innerhalb dieses Ereignisnetzes navigieren und auf einzelne Abschnitte dieser Handlung zugreifen und diese unterschiedlich vertexten kann - und so die Leser entsprechend seiner Intention zu lenken vermag.

\section{Ausblick}

Nachdem die exemplarische Diskussion von Röm 15,18-32 die Bedeutung von futurischen Ereignis-Bezügen als Fragmente potenzieller Erzählungen aufgezeigt hat, soll unten (Kapitel 13 und 14) ein ausführlicher Überblick über verschiedene sprachliche Mittel gegeben werden, mit denen solche Erzählfragmente an die

109 Zur Unterscheidung dominanter und subsidiärer Illokutionen vgl. ebenfalls oben, Kapitel 8, Abschnitt 4.4.3.

110 Vgl. etwa Bird, Romans, 502: „Paul's goal is to inform the Romans of his missionary work to date and how it relates to his plans to travel to Jerusalem, Rome, and Spain.“ 
Textoberfläche treten können. Für die erfolgreiche Rekonstruktion von paulinischen Protonarrativen ist es unerlässlich, dass diese Phänomene in ihrer Gesamtheit wahrgenommen und in ihrer Verschiedenheit berücksichtigt werden. Denn nicht jeder Verweis auf Zukünftiges hat dieselbe Funktion für ein damit eventuell verbundenes Protonarrativ.

Die Darstellung ist bewusst ausführlich gewählt, um herauszuarbeiten, dass es sich hierbei tatsächlich um ein im corpus Paulinum durchgängig belegtes, aus narratologischer Sicht wahrzunehmendes Element handelt. Die Materialfülle erlaubt allerdings nicht immer eine sämtliche Belege abdeckende Diskussion, wobei dann auf die Einschränkung der Datenbasis hingewiesen wird. Auch nur ansatzweise kann auf eventuelle Entwicklungslinien und das Verhältnis der unumstrittenen und umstrittenen Paulusbriefe eingegangen werden, auch wenn hier durchaus relevante Vorarbeiten geleistet werden sollen. ${ }^{111}$

Aus Platzgründen kann die Integration dieser Zukunftsbezüge in einen größeren narrativen Rahmen außerdem in der Regel nicht mit derselben Ausführlichkeit erfolgen, wie dies für Röm 15,18-32 geschah. Es könnte zudem in vielen Fällen der Grad der dem Text zugrundeliegenden Narrativisierung und der Mehrgewinn für die Interpretation kritisch diskutiert werden. Anders als in der in diesem Abschnitt gebotenen Tiefenbohrung soll im später gebotenen Überblick jedoch auch nicht die Relevanz für die Deutung der individuellen Textabschnitte im Zentrum stehen. Der Schwerpunkt liegt auf einer möglichst umfassenden Darstellung der (a) Bandbreite der sprachlichen Phänomene, mit welchen in den Paulusbriefen grundsätzlich Zukunftsbezüge hergestellt werden können und (b) auf der Differenzierung der unterschiedlichen Effekte, die durch verschiedene Kategorien an Vorausdeutungen erzielt werden können. Die zusammengetragenen Einzelbeobachtungen zeigen also ein Potenzial für die Forschung auf, das es im Einzelnen noch exegetisch auszuwerten gilt.

Dieser sehr ausführliche Überblick stellt somit auch eine Anleitung dar, wie einzelne Schriften des Paulus in ihrer Gesamtheit unter Berücksichtigung von futurischen Ereignissen auf Protoerzählungen hin zu analysieren sind. Wo dies in der gebotenen Knappheit möglich ist, soll dieses heuristische Potenzial zumindest schlaglichtartig angedeutet und mit Positionen der exegetischen Literatur in Dialog gebracht werden. Den besagten Kapitel 13 und 14 geht nun aber zunächst das im Vergleich mit jenen mit ganz ähnlicher Zielsetzung verfasste Kapitel 12 voraus, welche die theoretischen Überlegungen aus Kapitel 10 zum Diserzählten aufgreift und an konkreten Texten expliziert.

111 Siehe grundsätzlich oben, Kapitel 4, Abschnitt 2. 
